\title{
Decision Aiding
}

\section{A visual interactive tolerance approach to sensitivity analysis in MOLP}

\author{
Ana Rosa Pereira Borges ${ }^{\mathrm{a}, \mathrm{c}, *}$, Carlos Henggeler Antunes ${ }^{\mathrm{b}, \mathrm{c}}$ \\ a ISEC, Coimbra Polytechnic Institute, Apartado 10057, Quinta da Nora, 3030-601 Coimbra, Portugal \\ ${ }^{\mathrm{b}}$ Department of Electrical Engineering, University of Coimbra, Polo II, 3030-030 Coimbra, Portugal \\ ' INESC, Rua Antero de Quental 199, 3000-033 Coimbra, Portugal
}

Received 11 January 2001; accepted 3 August 2001

\begin{abstract}
This paper deals with the tolerance approach to sensitivity analysis of the weights in multiple objective linear programming (MOLP) problems. A geometric interpretation to compute the results in Hansen et al. [Eur. J. Oper. Res. 38 (1989) 63-69] is presented. Along the same direction, a visual interactive approach based on the analysis of indifference regions corresponding to basic efficient solutions is described.

(c) 2002 Elsevier Science B.V. All rights reserved.
\end{abstract}

Keywords: Multiple objective linear programming; Sensitivity analysis; Tolerance approach; Decision analysis; Interactive methods

\section{Introduction}

Sensitivity analysis in mathematical programming problems is aimed at helping to deal with the uncertainty and imprecision which are inherent to the models. In linear programming it generally computes ranges to indicate how much given coefficients can change before the optimal basis changes. However, the ranges obtained by traditional sensitivity analysis are easily computed only when the coefficients are not allowed to change in a simultaneous manner. Wendell $(1984,1985)$ and Ravi and Wendell $(1985,1989)$ developed the tolerance approach that enables us to consider the impact of simultaneous and independent changes of more than one coefficient. This approach also enables the use of a priori information about the variability of the coefficients.

The tolerance approach to sensitivity analysis in multiple objective linear programming (MOLP) problems proposed by Hansen et al. (1989) is an extension of the tolerance approach in linear programming. It considers perturbations on the $p$ objective functions weights when using the weighted-sum

\footnotetext{
${ }^{*}$ Corresponding author. Tel.: +351-239-851040; fax: +351-239-824692.

E-mail address: arborges@isec.pt (A.R. Pereira Borges).
} 
approach to compute efficient solutions. In particular, it yields the largest percentage, called the maximum tolerance percentage $\left(\tau^{*}\right)$, by which all weights can deviate simultaneously and independently from their estimated values while retaining the same efficient basic solution. A set of hypercubes, centered on the estimated weights and with radius $\tau_{k}$ is then defined, $C\left(\tau_{k}\right)$, in which $k$ refers to each nonbasic variable. The maximum tolerance value is associated with the largest hypercube for which all points in the hypercube represent changes to the weights such that the efficient solution obtained with the estimated weights remains efficient. This hypercube represents the tolerance region for the weights. It is also considered a priori information about the variability of the weights, for the case where weights are known to vary within intervals, leading to larger tolerance percentage. Let $\Phi$ be a specified polyhedron within which the estimated weights are known to vary. If $\Phi \neq \mathbb{R}^{p}$, a set of convex geometric solids that correspond to the intersection of $C\left(\tau_{k}\right)$ with $\Phi$ is then studied in the Euclidean space of dimension $p$. As previously, the maximum tolerance value is associated with the largest geometric solid for which all points in it represent changes to the weights such that the efficient solution computed with the estimated weights remains efficient.

Recently, attention has been paid to the tolerance approach in MOLP (Mármol and Puerto, 1997). The authors develop results which enlarge the range of the meaningful regions for the weights that can be handled by the tolerance approach, that include those of Hansen et al. (1989) as particular cases.

The use of the decomposition of a $(p-1)$-dimensional simplex in the $p$-dimensional Euclidean weight space (defined by normalized weighting vectors such that their components add-up to one) in indifference regions in MOLP has already been proposed by Antunes and Clímaco (1992) in the framework of TRIMAP method (Clímaco and Antunes, 1987, 1989).

This paper is devoted to perform a geometric based analysis of indifference regions in the $(p-1)$ dimensional simplex in a $p$-dimensional Euclidean weight space, in order to develop interactive tools to exploit the impact of weight changes. The maximum tolerance of the objective function weights are computed, and the changes of the tolerance region for an efficient basic solution are dynamically visualized when the information on the weights changes.

This paper is organized as follows. In Section 2, the statement of the problem is made and the results presented in Hansen et al. (1989) are revisited using a geometric analysis. Section 3 describes the integration of the tolerance approach to sensitivity analysis with the decomposition of the $(p-1)$-dimensional simplex in the $p$ Euclidean weight space. The results are illustrated in Section 4. Finally, some conclusions are drawn in Section 5.

\section{A geometric interpretation of the approach of Hansen et al. (1989)}

A geometric interpretation is developed in Wendell $(1984,1985)$ for the problem of finding the maximum tolerance percentage of the objective function coefficients and right-hand sides (RHSs) in a single objective linear programming problem.

The results presented in Hansen et al. (1989) can also be interpreted via a geometric analysis of the critical and tolerance regions for the weights (in analogy with Wendell's definitions).

\subsection{Statement of the MOLP problem}

Let us consider the following MOLP problem with $p$ linear objective functions and $m$ linear constraints:

$$
\text { "max" } \mathbf{z}=C \mathbf{x}
$$

s.t.

$$
\mathbf{x} \in X=\left\{\mathbf{x} \in \mathbb{R}^{n}: A \mathbf{x}=\mathbf{b}, \mathbf{x} \geqslant \mathbf{0}\right\}
$$


where $A$ is a $m \times n$ matrix, $\mathbf{b}$ is a $m$ column vector of the RHS terms and $C$ is a $p \times n$ matrix of objective functions coefficients. "max" denotes the operation of computing efficient solutions.

A feasible solution to (1) is called efficient if and only if there is no feasible solution for which no improvement in any objective functions value is possible without sacrificing on at least one of the other objective functions. A more relaxed notion is also used: a feasible solution is called weakly efficient if and only if there does not exist another feasible solution that strictly improves all objective function values.

Let $C_{r} .(r=1, \ldots, p)$ be the $r$ th row of $C$. When solving problem (1) by the weighted-sum approach, each objective $C_{r_{r}} \mathbf{x}$ is associated with a positive weight $\lambda_{r}$ (a $\lambda_{r}=0$ could lead to a weakly efficient solution) and efficient basic solutions can be obtained by solving the following weighted-sum problem:

$$
\max \sum_{r=1}^{p} \lambda_{r}\left(C_{r} \mathbf{x}\right)
$$

s.t.

$$
\mathbf{x} \in X, \quad \lambda_{r}>0, \quad r=1, \ldots, p .
$$

The tolerance approach to sensitivity analysis of the weight coefficients in (2) is developed through the following perturbed problem:

$$
\max \sum_{r=1}^{p}\left(\hat{\lambda}_{r}+\phi_{r} \lambda_{r}^{\prime}\right)\left(C_{r_{.}} \mathbf{x}\right)
$$

s.t.

$$
\mathbf{x} \in X, \quad \hat{\lambda}_{r}>0, \quad r=1, \ldots, p,
$$

where $\lambda_{r}^{\prime}>0, \hat{\lambda}_{r}$ represent the estimated value of $\lambda_{r}$ and $\phi_{r}$ are multiplicative parameters of $\lambda_{r}^{\prime}, r=1, \ldots, p$. Although theoretical results may be developed for any choice of $\lambda_{r}^{\prime}$, herein $\phi_{r}$ is considered as a percentage deviation from the estimated values (i.e. $\lambda_{r}^{\prime}=\hat{\lambda}_{r}$ ).

Let $K$ be the index set of the $n-m$ nonbasic variables associated with a basic efficient solution to (1), computed by using a given set of estimated weights, $\hat{\lambda}=\left(\hat{\lambda}_{1}, \ldots, \hat{\lambda}_{r}, \ldots, \hat{\lambda}_{p}\right)$, to solve (2). In order to be also an optimal solution to (2) or (3), the basic solution must satisfy the condition $(4 . k)$ or $\left(4^{\prime} . k\right)$, respectively

$$
\begin{aligned}
& \sum_{r=1}^{p} \lambda_{r} w_{r k} \geqslant 0, \quad k \in K, \\
& \sum_{r=1}^{p} \hat{\lambda}_{r} w_{r k}+\sum_{r=1}^{p} \phi_{r} \lambda_{r}^{\prime} w_{r k} \geqslant 0, \quad k \in K,
\end{aligned}
$$

in which $w_{r k}$ is the $(r, k)$ element of the reduced cost matrix of the multiobjective simplex tableau associated with the optimal solution to the weighted-sum scalar problem, with respect to objective function $r=1, \ldots, p$, and the nonbasic variable $k \in K$.

Therefore, the critical region for the weights corresponding to an efficient solution to (1), $H$, can be defined by

$$
H=\bigcap_{k \in K}\left\{\sum_{r=1}^{p} \hat{\lambda}_{r} w_{r k}+\sum_{r=1}^{p} \phi_{r} \lambda_{r}^{\prime} w_{r k} \geqslant 0\right\} .
$$

A nonnegative number $\tau$ is called an allowable tolerance for the weight perturbation if and only if the same basis $B$ is optimal to (3) as long as $\phi=\left(\phi_{1}, \ldots, \phi_{r}, \ldots, \phi_{p}\right) \in \Phi$ and $\|\phi\|_{\infty} \leqslant \tau$, where $\|\phi\|_{\infty}=$ $\mathrm{x}\left\{\max \left|\phi_{r}\right|, r=1, \ldots, p\right\}$ is the Tchebycheff norm of $\phi$ (the absolute value of each perturbation coefficient 
$\phi_{r}$ does not exceed $\left.\tau\right) . C(\tau)$ is the hyperbox in the weight space for which all points represent changes to the weights such that the corresponding efficient solution to (1) remains efficient:

$$
C(\tau)=\left\{\phi \in \mathbb{R}^{p}:\|\phi\|_{\infty} \leqslant \tau\right\} .
$$

This is a hypercube centered on the estimated weights with radius $\tau$.

Hansen et al. (1989) calculate the maximum tolerance percentage of the objective functions weights, $\tau^{*}$, for which the corresponding hyperbox $C\left(\tau^{*}\right)$ on the weight space is a subset of the region $H$ obtained with an estimated set of weights $\hat{\lambda}$. If there are additional constraints imposed by the decision maker (DM) (that is, a priori information about the variability of the weights, such that $\Phi \neq \mathbb{R}^{p}$ ) then $\tau^{*}$ is the maximum tolerance percentage for which the intersection of $\Phi$ with the corresponding hyperbox $C\left(\tau^{*}\right)$ in the weight space is a subset of the region $H$, that is:

$$
C\left(\tau^{*}\right) \cap \Phi \subseteq H .
$$

If $\Phi=\mathbb{R}^{p}$ then the hyperbox centered on the estimated weights $\hat{\lambda}$ has one of its vertexes belonging to one of the hyperplanes obtained from the $n-m$ constraints in $\left(4^{\prime} . k\right)$ replacing the inequality ' $\geqslant$ ' by ' $=$ '. Consider a case with $p=2$ and $n-m=2$ which is illustrated in Fig. 1. The boundaries of each hyperbox (in this example, the hyperboxes are rectangles) and the two halfspaces $(k=1,2)$ are also displayed. Point $\mathrm{A}$ is the vertex belonging to a hyperplane that corresponds to the largest rectangle within both halfspaces.

If the DM is able to specify a priori additional constraints with respect to the range of variability of the estimated weights $\hat{\lambda}\left(\Phi \neq \mathbb{R}^{p}\right)$, the vertex belonging to the hyperplane (obtained from the corresponding constraint) is a vertex of the region obtained by the intersection of those additional constraints with the hyperbox $C(\tau)$. Point $B$ in Fig. 2(a) ( $p=2$ and $n-m=1$, for the sake of readability) is the vertex of the

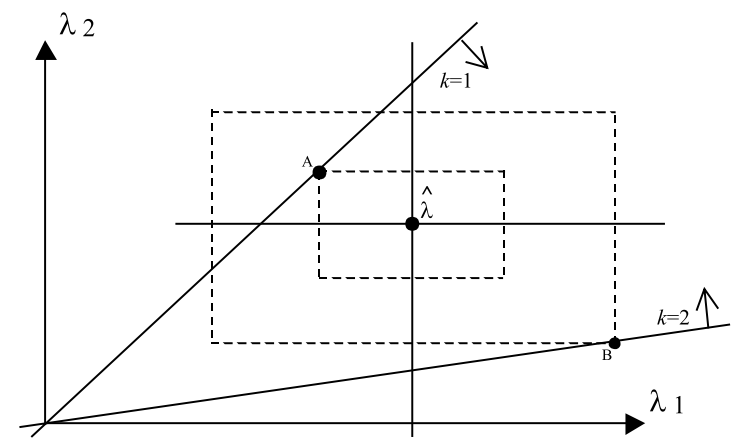

Fig. 1.

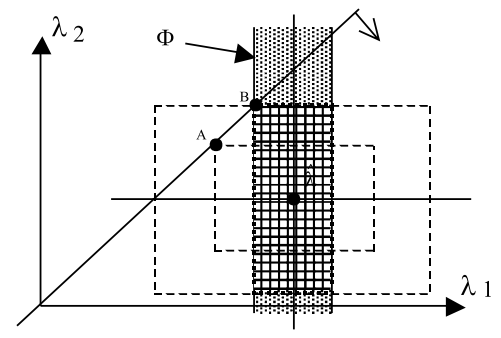

(a)

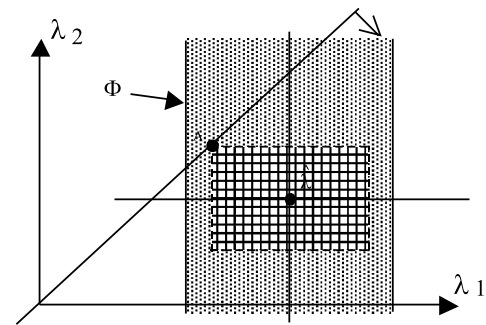

(b)

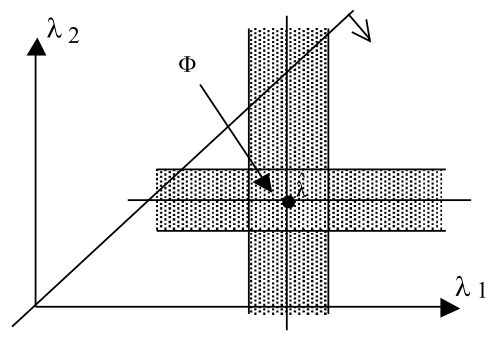

(c)

Fig. 2. 
hatched rectangle that is obtained by the intersection of $\Phi$ with the hyperbox (rectangle with point $B$ in the boundary).

In Fig. 2(b) the a priori additional information about the range of variability of the weights does not affect the obtained result with $\Phi=\mathbb{R}^{2}$ (the a priori variability range of weights is greater than the obtained considering the maximum tolerance percentage). In Fig. 2(c), $\tau^{*}$ is not finite.

\subsection{No a priori information}

If no a priori additional information about the variability of the objective function weights is specified then $\Phi=\mathbb{R}^{p}$.

For each of the $n-m$ constraints of $\left(4^{\prime} . k\right)$ the largest hyperbox centerd on $\hat{\lambda}=\left(\hat{\lambda}_{1}, \ldots, \hat{\lambda}_{r}, \ldots, \hat{\lambda}_{p}\right)$ that belong to the hyperhalfspace defined by the corresponding constraint in $\left(4^{\prime} . k\right)$ must be found.

The vertex of each hyperbox $(k=1, \ldots, n-m)$ can be characterized by $\left(\hat{\lambda}_{1}( \pm) \phi_{1} \lambda_{1}^{\prime}, \ldots, \hat{\lambda}_{r}( \pm)\right.$ $\left.\phi_{r} \lambda_{r}^{\prime}, \ldots, \hat{\lambda}_{p}( \pm) \phi_{p} \lambda_{p}^{\prime}\right)$, where $\phi_{r}$ is the perturbation coefficient of the estimated weight $\hat{\lambda}_{r}, r=1, \ldots, p$. The vertex that in each hyperbox belongs to the hyperplane obtained from the corresponding constraint in $\left(4^{\prime} . k\right)$ (replacing the inequality ' $\geqslant$ ' by ' $=$ ') can be characterized by $Q=\left(\hat{\lambda}_{1}-s_{1} \phi_{1} \lambda_{1}^{\prime}, \ldots, \hat{\lambda}_{r}-s_{r} \phi_{r} \lambda_{r}^{\prime}, \ldots\right.$, $\left.\hat{\lambda}_{p}-s_{p} \phi_{p} \lambda_{p}^{\prime}\right)$, where $s_{r}=\operatorname{sgn}\left[\lambda_{r}^{\prime} w_{r k}\right], r=1, \ldots, p$.

The hyperbox satisfying all the constraints that define region $H$ on the weight space for the estimated weights under analysis is the smallest of all the $n-m$ hyperboxes found, and the maximum tolerance $\tau^{*}$ is the smallest of all obtained $\tau_{k}$ values $(k=1, \ldots, n-m)$.

Let us begin by geometrically studying the case when the hyperbox does not exist, for which Hansen et al. (1989) consider $\tau_{k}$ is not finite. If no vertex belonging to the hyperplane associated with the $k$ constraint of $\left(4^{\prime} . k\right)$ could be found then the hyperbox does not exist. This situation happens if the constraint does not define a hyperhalfspace, that is, when all the coefficients are zero.

As noted by Wondolowski (1991), with respect to the Wendell's approach, this situation is associated with dual degeneracy and the value of $\tau_{k}$ shall be considered zero instead of infinite.

If some of the estimated weights are known with precision, the hyperbox can also not exist. This happens when the zero coefficients in $\left(4^{\prime} . k\right)$ are associated with precise estimated weights $\left(\lambda_{r}^{\prime}=0\right)$, that is, when the hyperplane obtained with precise weights is parallel to the hyperplane obtained from the corresponding constraint in $\left(4^{\prime} . k\right)$.

Note that these situations are in conformity with Corollary 2.2 in Hansen et al. (1989), that is if $\Phi=\mathbb{R}^{p}$ then $\tau_{k}=+\infty$ if and only if $\lambda_{r}^{\prime} w_{r k}=0$, for each $r=1, \ldots, p$.

Provided that, for each of the $n-m$ constraints obtained from the reduced cost matrix, the estimated weights and the vertex of the hyperbox belonging to the hyperplane under study are known, then the distance between these two points can easily be determined. Therefore, for each of the $n-m$ constraints, the distance from the point $\hat{\lambda}=\left(\hat{\lambda}_{1}, \ldots, \hat{\lambda}_{r}, \ldots, \hat{\lambda}_{p}\right)$ to the corresponding hyperplane is computed along the direction of the vector $\vec{V}=\left(-s_{1} \phi_{1} \lambda_{1}^{\prime}, \ldots,-s_{r} \phi_{r} \lambda_{r}^{\prime}, \ldots,-s_{p} \phi_{p} \lambda_{p}^{\prime}\right)$. The $\tau_{k}$ values $(k=1, \ldots, n-m)$ are obtained from these distances (and proportionally change with them).

Since the hyperbox satisfying all the constraints that define region $H$ for the estimated weights under analysis is the smallest of all $n-m$ hyperboxes, the $\tau^{*}$ value is also the smallest of all the determined $\tau_{k}$, $k=1, \ldots, n-m$.

Let us show how to calculate those distances and the corresponding $\tau_{k}$ values when the number of objective functions is $p=3$.

Fig. 3 shows a 3 -dimensional weight space where $P=\hat{\lambda}=\left(\hat{\lambda}_{1}, \hat{\lambda}_{2}, \hat{\lambda}_{3}\right)$ is the point corresponding to the estimated weights. The plane $\Pi_{k}$ is one of the planes obtained from the reduced cost matrix of the multiobjective simplex tableau corresponding to a basic efficient solution calculated by using the estimated weights $P$ and it is defined by $\sum_{r=1}^{3} \hat{\lambda}_{r} w_{r k}=0$ ( $\Pi_{k}$ contains the origin). $\overline{P X}$ equals the perpendicular distance from $P$ to the plane $\Pi_{k}$ and it is determined by 


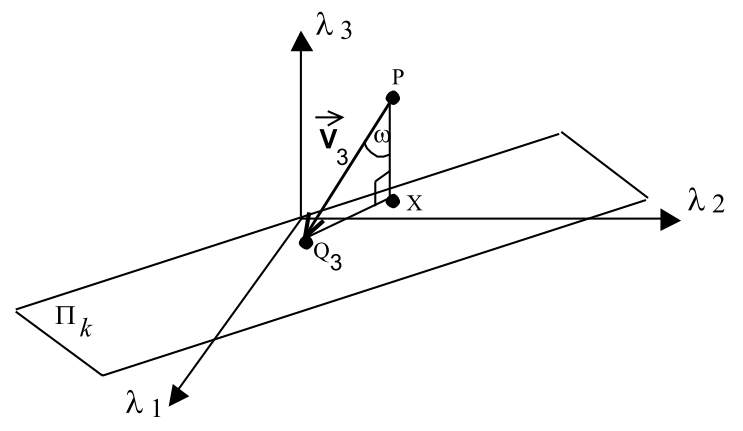

Fig. 3.

$$
\overline{P X}=\frac{\sum_{r=1}^{3} \hat{\lambda}_{r} w_{r k}}{\sqrt{\sum_{r=1}^{3} w_{r k}^{2}}} .
$$

The numerator absolute value sign is dropped due to the optimality of the unperturbed basic efficient solution.

$\overline{P Q_{3}}$ is the distance from $P$ to $\Pi_{k}$ measured along the direction of the vector $\vec{V}_{3}=\left(-s_{1} \tau_{k} \lambda_{1}^{\prime},-s_{2} \tau_{k} \lambda_{2}^{\prime}\right.$, $\left.-s_{3} \tau_{k} \lambda_{3}^{\prime}\right)$ and it is the distance we seek. Since $Q_{3}=\left(\hat{\lambda}_{1}-s_{1} \tau_{k} \lambda_{1}^{\prime}, \hat{\lambda}_{2}-s_{2} \tau_{k} \lambda_{2}^{\prime}, \hat{\lambda}_{3}-s_{3} \tau_{k} \lambda_{3}^{\prime}\right), \overline{P Q}_{3}=\left|\vec{V}_{3}\right|=$ $\tau_{k} \sqrt{\sum_{r=1}^{3} \lambda_{r}^{\prime 2}}$

Let $\omega$ be the angle between $[P, X]$ and $\left[P, Q_{3}\right]$, such that $\overline{P Q}_{3}=\overline{P X} / \cos \omega$.

Since $[P, X]$ is perpendicular to $\Pi_{k}$, it is parallel to each normal vector to $\Pi_{k}$, for instance to $\vec{N}=\left(-w_{1 k},-w_{2 k},-w_{3 k}\right)$ and $\cos \omega$ can be obtained from the dot-product of $\vec{V}_{3}$ and $\vec{N}$, that is,

$$
\cos \omega=\frac{\vec{V}_{3} \cdot \vec{N}}{\left|\vec{V}_{3}\right||\vec{N}|}=\frac{\tau_{k} \sum_{r=1}^{3}\left(-s_{r} \lambda_{r}^{\prime}\right)\left(-w_{r k}\right)}{\overline{P Q_{3}} \sqrt{\sum_{r=1}^{3} w_{r k}^{2}}} .
$$

Since $\overline{P Q_{3}}=\overline{P X} / \cos \omega$, the corresponding $\tau_{k}$ value can be determined from

$$
\begin{aligned}
& \overline{P Q_{3}}=\frac{\sum_{r=1}^{3} \hat{\lambda}_{r} w_{r k}}{\sqrt{\sum_{r=1}^{3} w_{r k}^{2}} \frac{\overline{P Q_{3}} \sqrt{\sum_{r=1}^{3} w_{r k}^{2}}}{\tau_{k} \sum_{r=1}^{3}\left(-s_{r} \lambda_{r}^{\prime}\right)\left(-w_{r k}\right)},} \\
& \tau_{k}=\frac{\sum_{r=1}^{3} \hat{\lambda}_{r} w_{r k}}{\sum_{r=1}^{3}\left(-s_{r} \lambda_{r}^{\prime}\right)\left(-w_{r k}\right)}, \quad k=1, \ldots, n-m .
\end{aligned}
$$

Since we want to compute the smallest distance from the point to the plane, and the corresponding smallest $\tau_{k}$, then $s_{r}=\operatorname{sgn}\left(w_{r k}\right), r=1,2,3$, that is,

$$
\tau_{k}=\frac{\sum_{r=1}^{3} \hat{\lambda}_{r} w_{r k}}{\sum_{r=1}^{3} \lambda_{r}^{\prime}\left|w_{r k}\right|}, \quad k=1, \ldots, n-m,
$$

where $\tau_{k}=+\infty$ if the denominator is zero for any $k$.

If $\tau_{k}$ is finite then, for each $k=1, \ldots, n-m$, the $\phi_{r}^{*}$ values can be calculated by

$$
\phi_{r}^{*}=\tau_{k}\left(-\operatorname{sgn}\left[\lambda_{r}^{\prime} w_{r k}\right]\right)=\tau_{k} \operatorname{sgn}\left[\lambda_{r}^{\prime}\left(-w_{r k}\right)\right], \quad r=1,2,3 .
$$


The previous procedure can be generalized in a straightforward manner for $p>3$ and the following result, corresponding to Corollary 3.1 in Hansen et al. (1989), is obtained.

If $\Phi=\mathbb{R}^{p}$ then the value $\tau_{k}$, for $k=1, \ldots, n-m$ (either finite or infinite) is given by

$$
\tau_{k}=\frac{\sum_{r=1}^{p} \hat{\lambda}_{r} w_{r k}}{\sum_{r=1}^{p} \lambda_{r}^{\prime}\left|w_{r k}\right|}
$$

where $\tau_{k}=+\infty$ if the denominator is zero for any $k$.

If $\tau_{k}$ is finite then

$$
\phi_{r}^{*}=\tau_{k} \operatorname{sgn}\left[\lambda_{r}^{\prime}\left(-w_{r k}\right)\right], \quad r=1,2, \ldots, p .
$$

If some of the weight values are precise, all the procedure holds but the corresponding components in $\vec{V}$ become zero, and in $Q$ they equal the $\hat{\lambda}_{r}$ value (due to $\lambda_{r}^{\prime}=0$ ), that is,

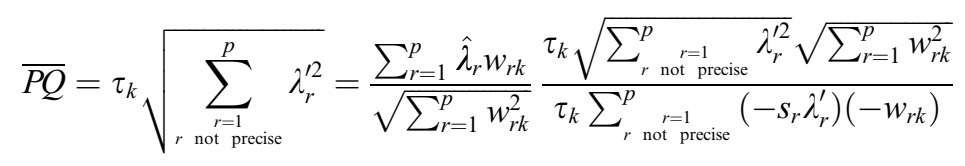

and

$$
\tau_{k}=\frac{\sum_{r=1}^{p} \hat{\lambda}_{r} w_{r k}}{\sum_{\substack{r=1 \\ r \text { not preise }}}^{p}\left(-S_{r} \lambda_{r}^{\prime}\right)\left(-w_{r k}\right)}, \quad k=1, \ldots, n-m .
$$

Since $\lambda_{r}^{\prime}=0$ when the corresponding weight is known with precision, we have Corollary 3.1 in Hansen et al. (1989) to determine the $\tau_{k}$ value.

\subsection{Weights are known to vary within intervals}

This section is devoted to perform a similar geometric analysis in the situation when the DM is able to specify some interval within which the values of the objective function weights are known to vary, $\underline{\lambda}_{r} \leqslant \hat{\lambda}_{r}+\phi_{r} \lambda_{r}^{\prime} \leqslant \bar{\lambda}_{r}, r=1, \ldots, p$. The interval $\left[\phi_{r}, \bar{\phi}_{r}\right]$ within which $\phi_{r}$ is allowed to vary is determined from the region $\left[\underline{\lambda}_{r}, \bar{\lambda}_{r}\right]$ and in this case $\Phi=\Phi(\underline{\phi}, \bar{\phi})$.

For $\lambda_{r}^{\prime}>0, \underline{\phi}_{r}$ and $\bar{\phi}_{r}$ can be computed as

$$
\underline{\phi}_{r}=\frac{\underline{\lambda}_{r}-\hat{\lambda}_{r}}{\lambda_{r}^{\prime}} \quad \text { and } \quad \bar{\phi}_{r}=\frac{\bar{\lambda}_{r}-\hat{\lambda}_{r}}{\lambda_{r}^{\prime}}
$$

In order to make $\left(\hat{\lambda}_{r}+\phi_{r} \lambda_{r}^{\prime}\right) \geqslant 0, \underline{\lambda}_{r}=0$ (or a small positive value to avoid the possibility of obtaining dominated solutions) is chosen such that $\phi_{r}=-\hat{\lambda}_{r} / \lambda_{r}^{\prime}$.

As it has been suggested by Wendell (1984) setting $\underline{\phi}_{r}=-\infty$ and $\bar{\phi}_{r}=+\infty$ means that there are no a priori bounds, and setting both values equal to zero $\overline{\mathrm{is}}^{r}$ an alternative way of specifying that the corresponding weight is a precise one.

For $\Phi=\Phi(\underline{\phi}, \bar{\phi})$ an iterative approach is proposed to determine $\tau_{k}$ (Hansen et al., 1989). After checking that $\tau_{k}$ is finite the problem is initially solved considering $\Phi=\mathbb{R}^{p}$ (unconstrained case), that is the perturbation coefficient is determined by using

$$
\phi_{r}^{0}=\operatorname{sgn}\left[\lambda_{r}^{\prime}\left(-w_{r k}\right)\right] \frac{\sum_{r=1}^{p} \hat{\lambda}_{r} w_{r k}}{\sum_{r=1}^{p} \lambda_{r}^{\prime}\left|w_{r k}\right|}, \quad r=1, \ldots, p .
$$

Afterwards, the finite limits $\underline{\phi}_{r}$ and $\bar{\phi}_{r}$ are gradually considered: in each iteration $t$ it is tested whether the obtained $\phi_{r}^{t}$ values satisfy the a priori constraints imposed by the DM (that is, $\phi^{t} \in \Phi(\underline{\phi}, \bar{\phi})$ ). If all 
constraints are satisfied then the current solution is optimal and the iterative process stops. Otherwise, those components $\phi_{r}^{t}$ that do not satisfy the constraints are fixed at values equal to the closest bounds. The remaining values are then recomputed after substitution of the fixed values in the considered hyperplane equation corresponding to $\left(4^{\prime} . k\right)$.

In each iteration $t>0$ one wants to determine the largest hyperbox for which its intersection with the hyperhalfspaces $\hat{\lambda}_{r} \geqslant \underline{\lambda}_{r}$ and $\hat{\lambda}_{r} \leqslant \bar{\lambda}_{r}$, for some $r=1, \ldots, p$, where the components $\phi_{r}^{t}$ of $\phi^{t}$ are not satisfied in an earlier iteration, results in a hyperbox that belongs to the hyperhalfspace corresponding to the $k$ equation of $\left(4^{\prime} . k\right)$. Let $\Phi^{t}$ denote the intersection of the hyperhalfspaces $\hat{\lambda}_{r} \geqslant \underline{\lambda}_{r}$ and $\hat{\lambda}_{r} \leqslant \bar{\lambda}_{r}$, defined in $\Phi$, for the $r=1, \ldots, p$ corresponding to the $\phi_{r}^{t}$ which do not satisfy $\underline{\lambda}_{r} \leqslant \hat{\lambda}_{r}+\phi_{r}^{t} \lambda_{r}^{\prime} \leqslant \bar{\lambda}_{r}$ until iteration $t$. Let $\Xi^{t}$ be the intersection of the hyperbox in iteration $t$, for which the vertexes are characterized by $\left(\hat{\lambda}_{1} \pm\right.$ $\left.\phi_{1}^{t} \lambda_{1}^{\prime}, \ldots, \hat{\lambda}_{p} \pm \phi_{p}^{t} \lambda_{p}^{\prime}\right)$, with $\Phi^{t}$.

Let us consider the example in Fig. 4(a) and (b) for $p=2$ and $n-m=2$. Two halfspaces $(k=1,2)$ as well as two different sets of a priori additional information, $\Phi$, are considered.

In all figures, $\Xi^{t}$ is the darkest rectangle and point $C$ is the vertex belonging to the hyperplane obtained from the corresponding constraint in $\left(4^{\prime} . k\right)$.

In Fig. 4(b) the constraints $\hat{\lambda}_{2} \geqslant \underline{\lambda}_{2}$ and $\hat{\lambda}_{2} \leqslant \bar{\lambda}_{2}$ are not considered because $\phi_{2}^{t}$ never violates the bounds $\underline{\phi}_{2}$ and $\bar{\phi}_{2}$.

In each iteration $t>0$, the vertex of $\Xi^{t}$ that belongs to the hyperplane, obtained from the corresponding constraint in $\left(4^{\prime} . k\right)$, has as co-ordinates the constant values $\hat{\lambda}_{r}+\phi_{r}^{t} \lambda_{r}^{\prime}$ in the components for which $\phi_{r}^{t}$ have not satisfied the constraint $\phi_{r}^{t} \geqslant \phi_{r}$ or $\phi_{r}^{t} \leqslant \bar{\phi}_{r}$ in an early iteration. For example, if the components $v$ and $d$ of $\phi^{t}$ have just violated the a priori bounds at iteration $t$, the vertex which is on the hyperplane can be characterized by

$$
Q^{\prime}=\left(\hat{\lambda}_{1}-s_{1} \phi_{1} \lambda_{1}^{\prime}, \ldots, \hat{\lambda}_{v}+\phi_{v}^{t} \lambda_{v}^{\prime}, \ldots, \hat{\lambda}_{r}-s_{r} \phi_{r} \lambda_{r}^{\prime}, \ldots, \hat{\lambda}_{d}+\phi_{d}^{t} \lambda_{d}^{\prime}, \ldots, \hat{\lambda}_{p}-s_{p} \phi_{p} \lambda_{p}^{\prime}\right),
$$

where $\phi_{r}$ is the perturbation coefficient of the estimated weight $\lambda_{r}^{\prime}$ and $s_{r}=\operatorname{sgn}\left[\lambda_{r}^{\prime} w_{r k}\right], r=1, \ldots, p, r \neq v$ and $r \neq d$.

When all components of $\phi_{r}^{t+1}, r=1,2, \ldots, p$, calculated in iteration $t$ satisfy the a priori bounds $\left(\phi_{r}\right.$ and $\bar{\phi}_{r}$ ), the iterative process ends and the corresponding $\tau_{k}$ value is the greatest absolute value of $\phi_{r}^{t+1}$.

The hyperbox for which $\Xi^{t}$ satisfy all the constraints that define region $H$ on the weight space for the estimated weights under consideration is selected. This is the smallest of all the $n-m$ hyperboxes found. In the examples of Fig. 4(a) and (b) the smallest hyperbox is obtained for $k=1$.

The maximum tolerance percentage of the objective functions weights, $\tau^{*}$, is the smallest of the $\tau_{k}$ values obtained, $k=1, \ldots, n-m$ (as in Section 2.2).

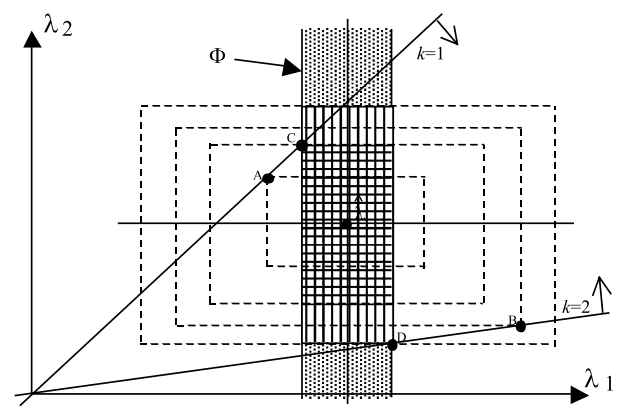

(a)

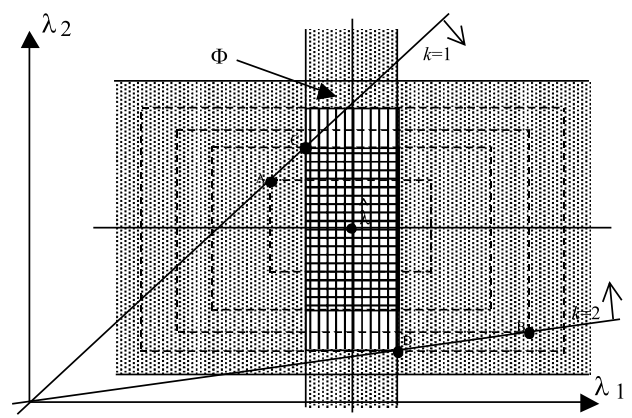

(b)

Fig. 4. 
Since the $\tau_{k}$ value cannot decrease when introducing additional information, the following more efficient procedure to determine $\tau^{*}$ can be recommended (Mármol and Puerto, 1997):

(1) For those indices $k$ for which $\tau_{k}$ is finite, the $\tau_{k}$ values are initially computed by using (18.k) (for the unconstrained case).

(2) For the index $h$ for which the minimum value $\left(\tau_{h}\right)$ is attained, it is checked whether the perturbed weights are included in $\Phi$ (that is, they satisfy the hyperhalfspaces $\hat{\lambda}_{r} \geqslant \underline{\lambda}_{r}$ and $\hat{\lambda}_{r} \leqslant \bar{\lambda}_{r}$ for $r=$ $1, \ldots, p)$. In this case, this value is the maximum tolerance percentage $\tau^{*}=\tau_{h}$, otherwise the objective function relaxation procedure proposed in Hansen et al. (1989) must be used to recompute $\tau_{h}$.

Then all indices whose current values for $\tau_{k}$ are strictly lower than the computed value for $\tau_{h}$ are considered. If there are no such indices then the maximum tolerance percentage $\tau^{*}=\tau_{h}$ has been obtained.

Otherwise step (2) is repeated considering those indices only.

Let us begin by geometrically studying the case $\tau_{k}$ is not finite, $k=1, \ldots, n-m$.

From Fig. 2(c) $(p=2)$ it can be concluded that if the region defined by $\Phi$ is completely included in the halfspace defined by the $k$ constraint of $\left(4^{\prime} . k\right)$ then any value can be given for the estimated weights perturbation coefficient $\phi_{r}, r=1,2$, viz. $+\infty$, so $\tau_{k}=+\infty$. The generalization of this result for any dimension $p>2$ is straightforward: if region $\Phi$ is completely included in the hyperhalfspace defined by the $k$ constraint of $\left(4^{\prime} . k\right)$ then the coefficient $\phi_{r}, r=1, \ldots, p$, can take any value and therefore $\tau_{k}=+\infty$. The region defined by $\Phi$ is completely included in the halfplane $k$ under study whenever the vertex of $\Phi$ that is the closest to the halfray defined by the $k$ constraint of $\left(4^{\prime} . k\right)$ belongs to this halfplane (because if this vertex belongs to it, then the same applies to the whole region $\Phi$ ). The components of this point can take the value $\hat{\lambda}_{r}+\bar{\phi}_{r} \lambda_{r}^{\prime}$ if $\left(-w_{r k}\right)>0$ and $\hat{\lambda}_{r}+\underline{\phi}_{r} \lambda_{r}^{\prime}$ if $\left(-w_{r k}\right)<0, r=1,2$ (if $\left(-w_{r k}\right)=0$ then any value can be given to this component).

This result can be generalized to any dimension $p>2$, leading to Corollary 2.1 in Hansen et al. (1989).

Let us suppose that the DM specifies a priori additional constraints for all weights $\left(\hat{\lambda}_{r} \geqslant \underline{\lambda}_{r}\right.$ and $\hat{\lambda}_{r} \leqslant \bar{\lambda}_{r}$, for $r=1, \ldots, p)$. Geometrically, we can observe that if the $\tau_{k}$ value is finite then there is at least one dimension $(r=1, \ldots, p)$ for which these constraints are not violated. If all additional constraints are violated then $\Xi^{t}$ never intersects the hyperplane obtained from the $k$ constraint of $\left(4^{\prime} . k\right)$, replacing the inequality ' $\geqslant$ ' by ' $=$ ', and the corresponding $\tau_{k}$ is not finite.

Therefore, the $\tau_{k}$ value, when finite, can be determined by the objective function relaxation procedure proposed in Hansen et al. (1989) in at most $p-1$ iterations instead of $p+1$ as mentioned by the authors.

Since, in each iteration $t>0$ the estimated weights and the vertex of the $\Xi^{t}$ that belongs to the hyperplane under study are known, the distance between these two points can easily be determined. As previously, for each of the $n-m$ constraints, the distance from the point $\hat{\lambda}=\left(\hat{\lambda}_{1}, \ldots, \hat{\lambda}_{r}, \ldots, \hat{\lambda}_{p}\right)$ to the corresponding hyperplane can be calculated, through the direction of the vector $\vec{V}^{\prime}$ from $\hat{\lambda}$ to the vertex of $\Xi^{t}$ that belongs to the hyperplane $k$. The values of $\phi_{r}^{t+1}, r=1, \ldots, p$, determined in iteration $t$, can be obtained from these distances.

We will show how to calculate these distances and the corresponding $\phi_{r}^{t+1}$ values, $r=1, \ldots, p$, when the number of objective functions is $p=3$. The generalization to any dimension is straightforward.

As mentioned before, in each iteration $t>0$, the vertex of $\Xi^{t}$ that belongs to the hyperplane obtained from the $k$ constraint in $\left(4^{\prime} . k\right)$ has as components the constant values $\hat{\lambda}_{r}+\phi_{r}^{t} \lambda_{r}^{\prime}$ if $\phi_{r}^{t}$ have not satisfied the constraint $\phi_{r}^{t} \geqslant \phi_{r}$ or $\phi_{r}^{t} \leqslant \bar{\phi}_{r}$ in a previous iteration.

Without loss of generality, let us consider $t=0$ and that the second component of $\phi^{t}$ violates one of the a priori bounds $\left(\phi_{r}\right.$ or $\left.\bar{\phi}_{r}\right)$. That is, $J^{t+1}=\{1,3\}, \phi_{2}^{t+1}=\left\{\phi_{2}\right.$ or $\left.\bar{\phi}_{2}\right\}$ (in accordance with the bound that is violated). The vertex belonging to the hyperplane under study is the point $Q_{3}^{\prime}=\left(\hat{\lambda}_{1}-s_{1} \tau \lambda_{1}^{\prime}, \hat{\lambda}_{2}+\phi_{2}^{t+1} \lambda_{2}^{\prime}\right.$, $\left.\hat{\lambda}_{3}-s_{3} \tau \lambda_{3}^{\prime}\right), s_{r}=\operatorname{sgn}\left[\lambda_{r}^{\prime} w_{r k}\right], r=1,3$, and the nonnegative number $\tau$ is an allowable tolerance for the weight perturbation.

$\overline{P Q_{3}^{\prime}}$ is the distance from $P$ to $\Pi_{k}$ measured through the direction of the vector $\vec{V}_{3}^{\prime}=\left(-s_{1} \tau \lambda_{1}^{\prime}, \phi_{2}^{t+1} \lambda_{2}^{\prime}\right.$, $\left.-S_{3} \tau \lambda_{3}^{\prime}\right)$, which is the distance to be computed. $\overline{P Q_{3}^{\prime}}=\left|\vec{V}_{3}^{\prime}\right|$ and 


$$
\overline{P Q_{3}^{\prime}}=\tau \sqrt{\sum_{\substack{r=1 \\ r \in J^{t+1}}}^{3} \lambda_{r}^{\prime 2}}+\sqrt{\sum_{\substack{r=1 \\ r \notin J^{t+1}}}^{3}\left(\phi_{r}^{t+1} \lambda_{r}^{\prime}\right)^{2}}=\tau \sqrt{\sum_{\substack{r=1 \\ r \neq 2}}^{3} \lambda_{r}^{\prime 2}}+\phi_{2}^{t+1} \lambda_{2}^{\prime} .
$$

If $\omega$ is the angle between $[P, X]$ and $\left[P, Q_{3}^{\prime}\right]$ then $\overline{P Q_{3}^{\prime}}=\overline{P X} / \cos \omega$ and

$$
\cos \omega=\frac{\vec{V}_{3}^{\prime} \cdot \vec{N}}{\left|\vec{V}_{3}^{\prime}\right||\vec{N}|}=\frac{\tau \sum_{r \in J^{t+1}}^{3}\left(-s_{r} \lambda_{r}^{\prime}\right)\left(-w_{r k}\right)+\sum_{\substack{r=1 \\ r \neq t+1}}^{3}\left(\phi_{r}^{t+1} \lambda_{r}^{\prime}\right)\left(-w_{r k}\right)}{\overline{P Q_{3}^{\prime}} \sqrt{\sum_{r=1}^{3} w_{r k}^{2}}},
$$

where $\vec{N}=\left(-w_{1 k},-w_{2 k},-w_{3 k}\right)$.

From (8.k) we have

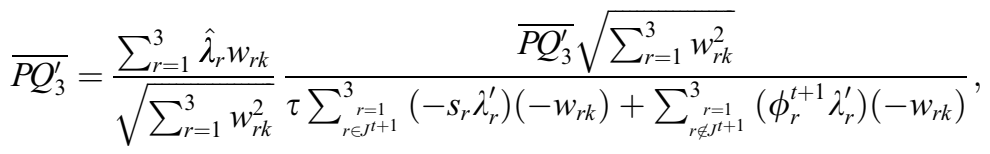

$$
\begin{aligned}
& \tau=\frac{\sum_{r=1}^{3} \hat{\lambda}_{r} w_{r k}+\sum_{\substack{r=1 \\
r \notin J t+}}^{3}\left(\phi_{r}^{t+1} \lambda_{r}^{\prime}\right) w_{r k}}{\sum_{\substack{r=1 \\
r \in J^{t+1}}}^{3}\left(-S_{r} \lambda_{r}^{\prime}\right)\left(-w_{r k}\right)} .
\end{aligned}
$$

Since we want to determine the smallest distance from the point to the plane, and the corresponding the smallest $\tau$, we have $s_{r}=\operatorname{sgn}\left(w_{r k}\right), r=1,2,3$, that is,

$$
\tau=\frac{\sum_{r=1}^{3} \hat{\lambda}_{r} w_{r k}+\sum_{\substack{r=1 \\ r \notin t+1}}^{3}\left(\phi_{r}^{t+1} \lambda_{r}^{\prime}\right) w_{r k}}{\sum_{\substack{r=1 \\ r \in J^{t+1}}}^{3} \lambda_{r}^{\prime}\left|w_{r k}\right|}
$$

Since $\tau$ is finite then, for each $k=1, \ldots, n-m$, the $\phi_{r}^{t+1}$ values can be calculated by using

$$
\phi_{r}^{t+1}=\left(-\operatorname{sgn}\left[\lambda_{r}^{\prime} w_{r k}\right]\right) \tau=\operatorname{sgn}\left[\lambda_{r}^{\prime}\left(-w_{r k}\right)\right] \tau,
$$

that is,

$$
\phi_{r}^{t+1}=\operatorname{sgn}\left[\lambda_{r}^{\prime}\left(-w_{r k}\right)\right] \frac{\sum_{r=1}^{3} \hat{\lambda}_{r} w_{r k}+\sum_{\substack{r=1 \\ r \notin t+1}}^{3}\left(\phi_{r}^{t+1} \lambda_{r}^{\prime}\right) w_{r k}}{\sum_{\substack{r=1 \\ r \in J^{t+1}}}^{3} \lambda_{r}^{\prime}\left|w_{r k}\right|} .
$$

If the number of objective functions is $p>3$, the previous procedure can be generalized and the following result, corresponding to step 4 of the relaxation procedure when $r \in J^{t+1}$ in Hansen et al. (1989), is obtained:

$$
\phi_{r}^{t+1}=\operatorname{sgn}\left[\lambda_{r}^{\prime}\left(-w_{r k}\right)\right] \frac{\sum_{r=1}^{p} \hat{\lambda}_{r} w_{r k}+\sum_{\substack{r=1 \\ r \notin t+1}}^{p}\left(\phi_{r}^{t+1} \lambda_{r}^{\prime}\right) w_{r k}}{\sum_{\substack{r=1 \\ r \in J^{t+1}}}^{p} \lambda_{r}^{\prime}\left|w_{r k}\right|} .
$$

If some of the weight values are known with precision, the whole procedure holds.

This section has shown the geometric way of obtaining the results presented in Hansen et al. (1989) by calculating distances from the estimated weights to a point in each one of the $n-m$ hyperplanes obtained from $\left(4^{\prime} . k\right)$. As in Hansen et al. (1989), the Tchebycheff norm of $\phi$ and multiplicative perturbations of the 
weights have been considered. However, the analysis can also be applied for different norms and different choice of $\lambda_{r}^{\prime} \mathrm{s}$.

These geometric analyzes are further extended in Section 3 considering normalized weighting vectors in three objective linear problems. This approach is suited to be implemented as a visual interactive procedure that the DM can use as a tool to dynamically explore the impact of weight changes.

\section{A visual interactive tolerance approach to sensitivity analysis}

Without loss of generality, each weighting vector can be normalized so that its elements sum to one, that is, it is a point on the geometrical $(p-1)$-dimensional simplex in a $p$-dimensional Euclidean weight space:

$$
\Lambda=\left\{\lambda: \lambda \in \mathbb{R}^{p}, \sum_{r=1}^{p} \lambda_{r}=1, \lambda_{r}>0, r=1, \ldots, p\right\} .
$$

Basic efficient solutions to (1) can be computed by optimizing a scalarizing function consisting of a weighted sum of the objective functions.

For three objective functions the use of interactive graphical tools are particularly suited for the exchange of information with the DM.

The indifference regions for the weights (graphical display of the set of weighting vectors that leads to the same basic efficient solution) are defined in $\Lambda$. They are obtained by the intersection of the $n-m$ constraints resulting from the reduced cost matrix of a multiobjective simplex tableau associated with a basic efficient solution (see (4.k)). Therefore, the DM may be indifferent to all combinations of weighting vectors within it because they lead to the same efficient solution.

The hatched polygon shown in Fig. 5(a) and (b) is the indifference region associated with the basic efficient solution computed by optimizing the scalar weighted-sum LP with the weights $P$. Each of the $n-m$ $\left(n-m=4\right.$ in this example) halfspaces defined by $(4 . k)$ corresponds to a nonbasic variable. The plane $\Pi_{k}$ is obtained from equation $k$ of (4.k) replacing the inequality ' $\geqslant$ ' by ' $=$ '. $\pi_{k}$ denotes the intersection of $\Pi_{k}$ with $\Lambda$.

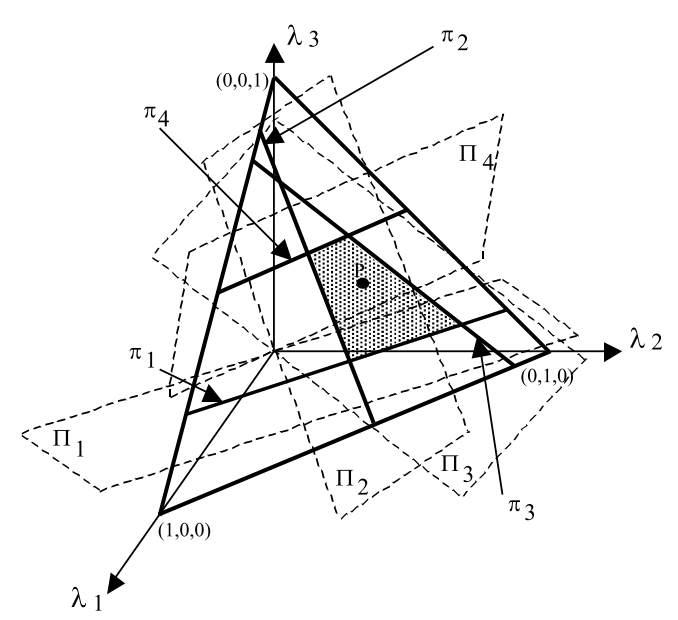

(a)

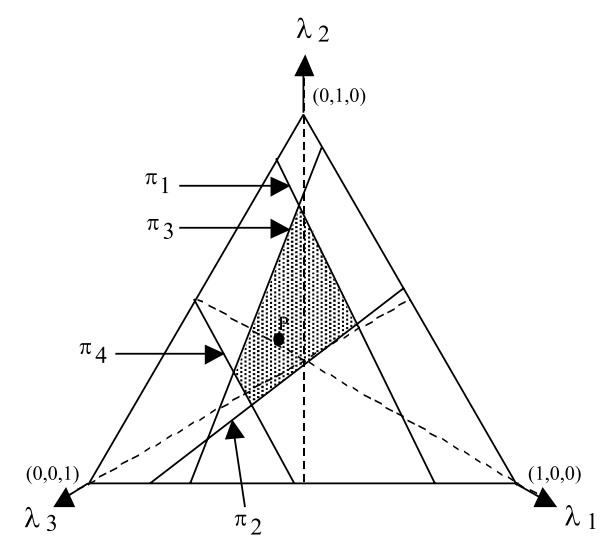

(b)

Fig. 5 . 


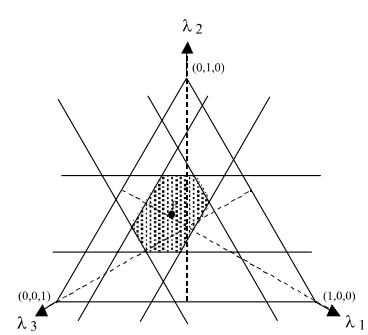

(a)

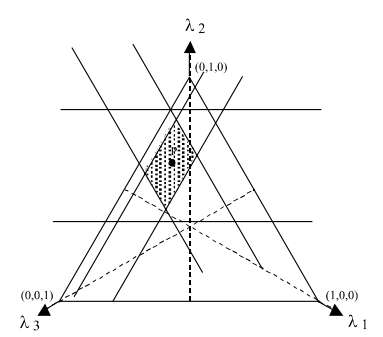

(b)

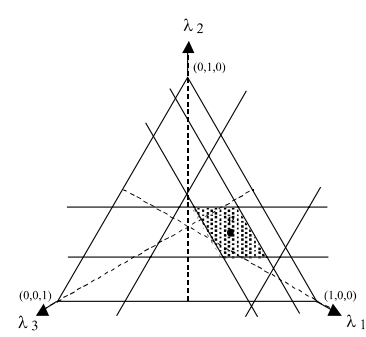

(c)

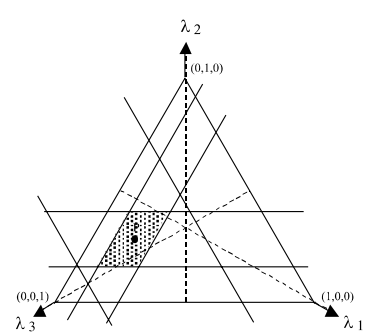

(d)

Fig. 6 .

The largest value for which all weights can deviate simultaneously and independently from their estimated values while retaining the same basic efficient solution in a MOLP problem can be determined by performing a geometric study analogous to the one in Section 2. However, the distortion resulting from the scaling condition of weights when computing the distances must be taken into account (see Evans, 1984; Schneller and Sphicas, 1985).

In this situation $C(\tau) \cap \Lambda$ are convex polygons as illustrated in Fig. 6(a)-(d). If $\Phi \neq \mathbb{R}^{3}$, the $C(\tau) \cap \Lambda \cap \Phi$ are as illustrated in Fig. 7(a) and (b).

Here we seek to calculate the maximum tolerance percentage of the objective function weights, $\tau^{*}$, for which the intersection of $\Lambda$ with the corresponding $C\left(\tau^{*}\right)$ is a subset of the region $H$ (see (5)) obtained with an estimated set of weights $P=\hat{\lambda}=\left(\hat{\lambda}_{1}, \hat{\lambda}_{2}, \hat{\lambda}_{3}\right)$. Or, if there are additional constraints imposed by the DM $\left(\Phi \neq \mathbb{R}^{3}\right), \tau^{*}$ for which the intersection of $\Phi$ with the corresponding $C\left(\tau^{*}\right) \cap \Lambda$ is a subset of the region $H$ obtained with the weights $\hat{\lambda}$. That is,

$$
C\left(\tau^{*}\right) \cap \Lambda \cap \Phi \subseteq H
$$

When $\Phi=\mathbb{R}^{3}$ one of the vertexes of $(C \tau)$ belongs to one of the $\pi_{k}$.

As Mármol and Puerto (1997) pointed out, even without information about weights these must always be considered nonnegative when dealing with efficient solutions. We agree with them but we also agree conceptually with Schneller and Sphicas (1985) for whom surely the DM is interested only in the possibility of reaching a nonoptimal basic efficient solution and therefore the weighting vector cannot "stray" out of $\Lambda$. We feel that both opinions are not totally in conflict. In fact, both authors consider that negative weights

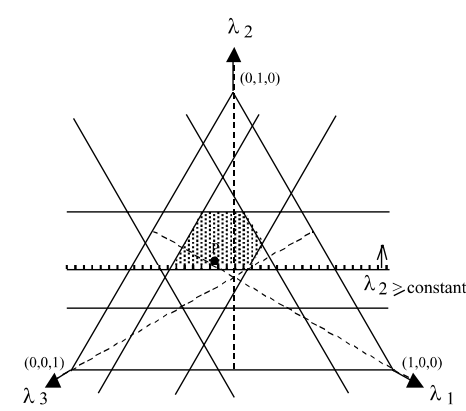

(a)

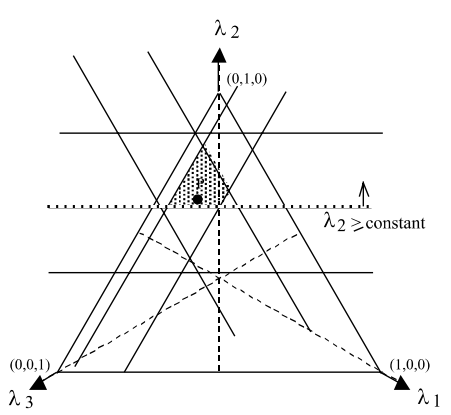

(b)

Fig. 7. 


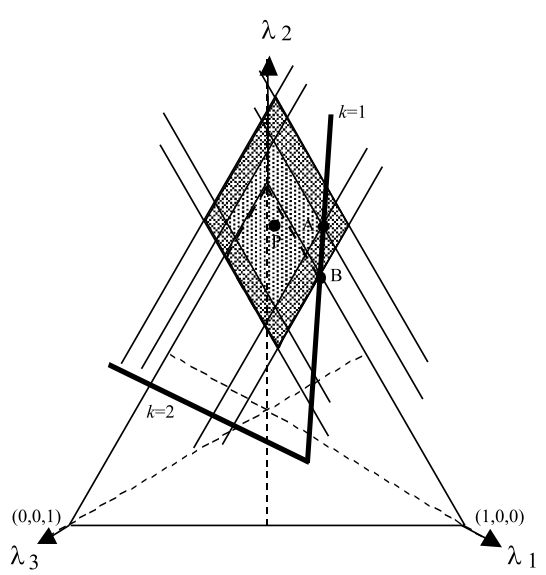

(a)

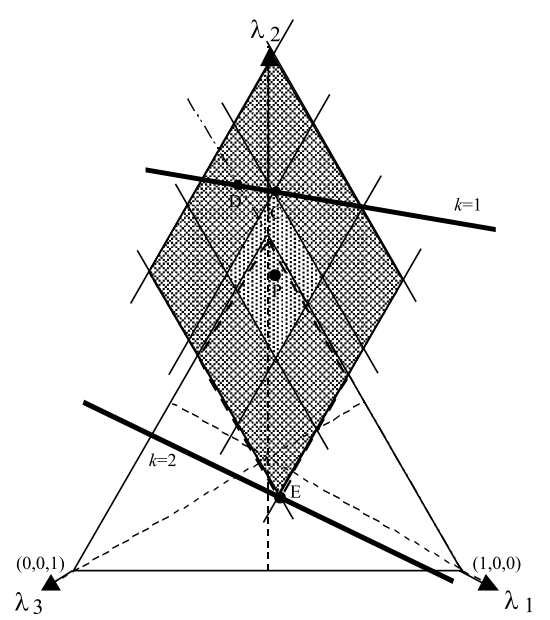

(b)

Fig. 8 .

have no meaning. In the operational framework of our visual interactive approach, the DM is only allowed to select positive weights. However, the considerations pointed out by both authors have been taken into account in the sense that it can happen $C\left(\tau^{*}\right)$ is not fully within $\Lambda$. In this case, the method guarantees that a larger tolerance percentage can be obtained, but related to positive weights. Therefore, if the vertex of $C(\tau)$ does not belong to $C(\tau) \cap \Lambda$ (some components of the vertex are negative), another $C(\tau)$ shall be computed such that one of the vertexes of $C(\tau) \cap \Lambda$ is the point that results from the intersection of $\pi_{k}$ with one of the planes $\lambda_{y}=0, y=1,2,3$ ( $y$ corresponding to the indices with negative component values in the vertex of the first computed $C(\tau)$ ), point $B$ in Fig. 8(a). If this point does not exist (that is, it lies outside $A$ ), the corresponding $\tau$ shall not be considered, point $D$ in Fig. 8(b).

If $\Phi \neq \mathbb{R}^{3}$, the vertex belonging to $\pi_{k}$ can also be a vertex of $C(\tau) \cap \Phi$. If this point does not belong to $C(\tau) \cap \Phi \cap \Lambda$, then one of the vertexes of $C(\tau) \cap \Phi \cap \Lambda$ is the point that results from the intersection of the corresponding $\pi_{k}$ with one of the planes $\lambda_{y}=0, y=1,2,3$, such that the component $y$ of the vertex of (the first one computed) $C(\tau) \cap \Phi$ has negative value. As previously, if it lies outside $\Lambda$ then the corresponding $\tau$ shall not be considered.

Let us consider a case in which $n-m=2(k=1,2)$ as illustrated in Fig. 8(a) and (b). In this example $C(\tau)$ are lozenges defined by the planes $\lambda_{1} \geqslant \hat{\lambda}_{1}-\tau_{k} \lambda_{1}^{\prime}, \lambda_{1} \leqslant \hat{\lambda}_{1}+\tau_{k} \lambda_{1}^{\prime}, \lambda_{3} \geqslant \hat{\lambda}_{3}-\tau_{k} \lambda_{3}^{\prime}$ and $\lambda_{3} \hat{\lambda}_{3}+\tau_{k} \lambda_{3}^{\prime}$.

Considering the example in Fig. 8(a), the greatest value for the tolerance percentage of the objective function weights, $\tau^{*}$, is obtained for $k=1$ (because with $k=2$ the corresponding $C(\tau) \cap \Lambda$ does not belong to the region $H$ defined in (5)). The following cases are also illustrated:

(i) the lozenge for which the vertex $A$ (belonging to $\pi_{1}$ ) does not belong to $C(\tau) \cap \Lambda\left(\lambda_{3}<0\right)$;

(ii) the lozenge for which $C(\tau) \cap \Lambda$ has as a vertex the point $(B)$ that results from the intersection of $\pi_{1}$ with the plane $\lambda_{3}=0$, and the corresponding boundaries of $C(\tau) \cap \Lambda$.

Fig. 8(b) illustrates the situation for which the value of $\tau_{1}$ shall not be considered, because with $k=1$ the point that results from the intersection of $\pi_{1}$ with the plane $\lambda_{3}=0(D)$ lies outside $\Lambda$. The greatest value for the tolerance percentage of the objective function weights, $\tau^{*}$, is obtained for $k=2$. The following cases are also illustrated:

(iii) the lozenge for which the vertex $C$ (belonging to $\pi_{1}$ ) does not belong to $C(\tau) \cap \Lambda\left(\lambda_{3}<0\right)$;

(iv) the lozenge for which $C(\tau) \cap \Lambda$ has as a vertex the point $(E)$ that belongs to $\pi_{2}$ and is inside the region $H$ defined in (5). 


\subsection{No a priori information}

Let us consider that no a priori additional information about the variability of the objective function weights is specified $\left(\Phi=\mathbb{R}^{3}\right)$.

Any one of the 12 'extreme points' shown in Fig. 9 is candidate to a vertex of $C(\tau)$. All these points are defined by $\left(\hat{\lambda}_{1}( \pm) \phi_{1} \lambda_{1}^{\prime}, \hat{\lambda}_{2}( \pm) \phi_{2} \lambda_{2}^{\prime}, \hat{\lambda}_{3}( \pm) \phi_{3} \lambda_{3}^{\prime}\right)$, where $\phi_{r}$ is the perturbation coefficient of the estimated weight $\hat{\lambda}_{r}, r=1,2,3$, and satisfy the condition $\hat{\lambda}_{1}( \pm) \phi_{1} \lambda_{1}^{\prime}+\hat{\lambda}_{2}( \pm) \phi_{2} \lambda_{2}^{\prime}+\hat{\lambda}_{3}( \pm) \phi_{3} \lambda_{3}^{\prime}=1$.

The vertex that in each $C(\tau)$ belongs to $\pi_{k}$ can be characterized by

(a) $T_{3(\mathrm{a})}=\left(\hat{\lambda}_{1}-s_{1} \phi_{1} \lambda_{1}^{\prime}, \hat{\lambda}_{2}-s_{2} \phi_{2} \lambda_{2}^{\prime}, 1-\hat{\lambda}_{1}+s_{1} \phi_{1} \lambda_{1}^{\prime}-\hat{\lambda}_{2}+s_{2} \phi_{2} \lambda_{2}^{\prime}\right) \Rightarrow$ points $A, B, C$ and $D$ in Fig. 9;

(b) $T_{3(\mathrm{~b})}=\left(\hat{\lambda}_{1}-s_{1} \phi_{1} \lambda_{1}^{\prime}, 1-\hat{\lambda}_{1}+s_{1} \phi_{1} \lambda_{1}^{\prime}-\hat{\lambda}_{3}+s_{3} \phi_{3} \lambda_{3}^{\prime}, \hat{\lambda}_{3}-s_{3} \phi_{3} \lambda_{3}^{\prime}\right) \Rightarrow$ points $E, F, G$ and $H$ in Fig. 9;

(c) $T_{3(\mathrm{c})}=\left(1-\hat{\lambda}_{2}+s_{2} \phi_{2} \lambda_{2}^{\prime}-\hat{\lambda}_{3}+s_{3} \phi_{3} \lambda_{3}^{\prime}, \hat{\lambda}_{2}-s_{2} \phi_{2} \lambda_{2}^{\prime}, \hat{\lambda}_{3}-s_{3} \phi_{3} \lambda_{3}^{\prime}\right) \Rightarrow$ points $I, J, L$ and $M$ in Fig. 9 .

The $s_{r}$ value is associated with the vertex under study and the slope of $\pi_{k}(k=1, \ldots, n-m)$ being considered.

It is necessary to separately study the cases for which the vertex of $C(\tau)$ belonging to $\pi_{k}$ is in situation (a), (b) or (c).

In each situation $e\left(e=(\mathrm{a})\right.$, (b) and (c)), we need to look for the largest $C\left(\tau_{k e}\right)$ for which one of its vertexes belongs to $\pi_{k}$. The smallest of all the three $C\left(\tau_{k e}\right)$ obtained is the one such that the intersection of $\Lambda$ with the corresponding $C\left(\tau_{k e}\right)$ is a subset of region $H$ obtained with an estimated set of weights. So, $\tau^{*}$ is the smallest of all the three $\tau_{k e}$ values obtained.

In order to determine the largest $C\left(\tau_{k e}\right) \quad(e=(\mathrm{a}),(\mathrm{b})$ or (c)) we need to study each of the $n-m$ constraints of $\left(4^{\prime} . k\right)$. That is, for each of the $n-m$ constraints of $\left(4^{\prime} . k\right)$, the largest $C\left(\tau_{k e}\right)$ for which the intersection of $\Lambda$ with the corresponding $C\left(\tau_{k e}\right)$ belongs to the halfspace defined by the corresponding constraint in $\left(4^{\prime} . k\right)$ must be found. For a situation $e$, the $C\left(\tau_{k e}\right)$ satisfying all the constraints that define the indifference region associated with the estimated weights is the smallest of all the $n-m C\left(\tau_{k e}\right)$ found and the $\tau_{k e}$ value is the smallest of all $\tau_{k e}$ values obtained, $k=1, \ldots, n-m$.

It may happen that in some situation $e(e=(\mathrm{a}),(\mathrm{b})$ or (c) $) C\left(\tau_{k e}\right)$ 'does not exist'. For instance, if the extreme point which is candidate to a vertex of $C\left(\tau_{k e}\right)$ is not effectively an extreme point on the boundary of $C\left(\tau_{k e}\right)$ then the corresponding $C\left(\tau_{k e}\right)$ determined in that situation for the corresponding $k$ constraint shall not be considered (point $A$ in Fig. 10(c)).

If the vertex of $C\left(\tau_{k e}\right)$ belonging to $\pi_{k}$ does not belong to $\left(C \tau_{k e}\right) \cap \Lambda$, it means that some vertex components are negative. For each index $y$ associated with a negative component, another $C\left(\tau_{k e}\right)$ shall be

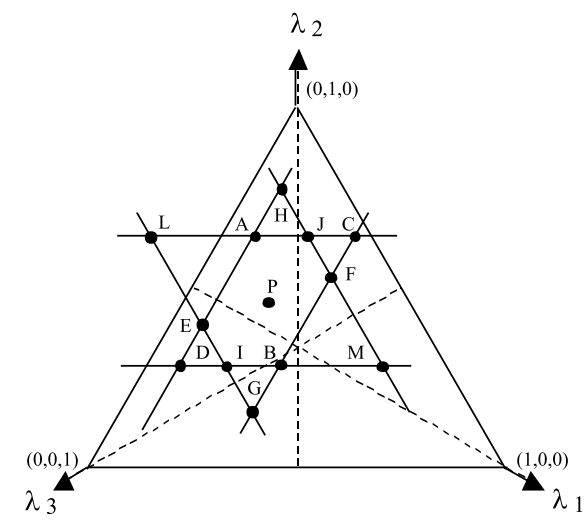

Fig. 9. 


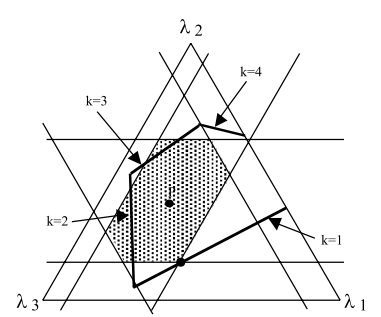

(a)

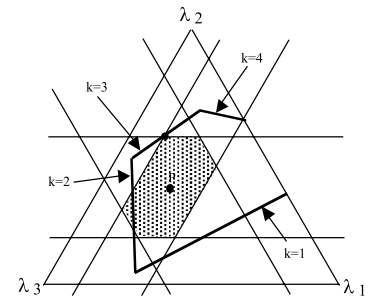

(b)

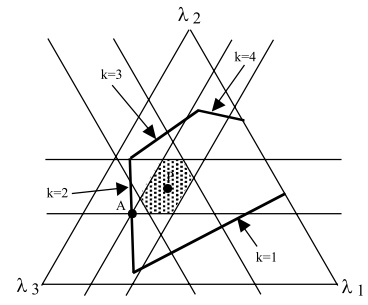

(c)

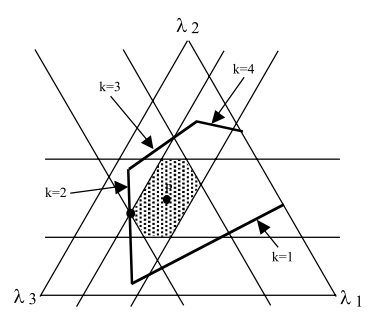

(d)

Fig. 10.

sought such that one of the vertexes of $C\left(\tau_{k e}\right) \cap \Lambda$ is the point that results from the intersection of $\pi_{k}$ with the plane $\lambda_{y}=0$. The smallest of all these $C\left(\tau_{k e}\right)$ obtained in this manner must be selected.

Since the $\tau_{k e}$ obtained is always greater than or equal to the initial $\tau_{k e}$ (with negative components) this procedure only needs to be performed if, for the smallest $\tau_{k e}$ obtained, the vertex of $C\left(\tau_{k e}\right)$ belonging to $\pi_{k}$ does not belong to $C\left(\tau_{k e}\right) \cap \Lambda$. For efficiency reasons, we recommend to compute initially all the $\tau_{k e}$ and, for the index $h$ where the minimum value $\tau_{k e}$ is attained, check whether the vertex of $C\left(\tau_{h e}\right)$ belonging to $\pi_{k}$ belongs to $C\left(\tau_{h e}\right) \cap \Lambda$. If so, this value is the maximum tolerance percentage; otherwise we must perform the previous procedure to recompute $\tau_{h e}$ and consider all the indices for which the current values of $\tau_{k e}$ are strictly less than the computed value for $\tau_{h e}$. If there are no such indices then we have obtained the maximum tolerance percentage $\tau_{k e}=\tau_{h e}$. Otherwise, those indices must also be studied (by means of a similar procedure to the one suggested in Section 2.3).

In Fig. 10(a)-(d) an indifference region associated with the basic efficient solution computed by using the estimated weights $P$ is shown.

If the extreme point of $C(\tau)$ belonging to $\pi_{k}$ is one of those referred to in situation (a), then for $k=1$ the region $C\left(\tau_{(1 \mathrm{a})}\right)$ of Fig. 10(a) is obtained, and for $k=3$ the region $C\left(\tau_{3(\mathrm{a})}\right)$ shown in Fig. 10(b) is computed. For $k=2$ (Fig. 10(d)) and $k=4$ the obtained extreme points are not effectively extreme points on the boundary of $C\left(\tau_{k(\mathrm{a})}\right)$. For situation (a) the smallest $C\left(\tau_{k(\mathrm{a})}\right)$ is obtained for $k=3$. Similar results can be determined when the extreme point of $C(\tau)$ belonging to the corresponding $\pi_{k}$ is one of those referred to in situations (b) and (c). The greatest $C(\tau)$ such that the intersection of $\Lambda$ with the corresponding $C(\tau)$ is a subset of the region $H$ obtained with an estimated set of weights $P$ is presented in Fig. 10(d) and it is associated with a vertex referred to in situation (b).

Geometrically it can be concluded that the $C\left(\tau_{k e}\right)$ corresponding to each one of the $n-m$ constraints of $\left(4^{\prime} . k\right)$ exists and the associated $\tau_{k e}$ value is finite if it is possible to find a vertex of $C\left(\tau_{k e}\right)$ that belongs to $\pi_{k}$, even if the obtained $C\left(\tau_{k e}\right)$ has a vertex that does not lie within $\Lambda$. That is, if $\Phi=\mathbb{R}^{3}$ then the $\tau_{k e}$ value is not finite if two of the estimated weights are defined with precision $\left(\lambda_{1}+\lambda_{2}+\lambda_{3}=1\right)$ or if the corresponding constraint $\left(4^{\prime} . k\right)$ does not define a halfplane (all the coefficients $w_{r k}$ are zero, for $r=1,2,3$ ).

In Hansen et al. (1989) the hyperplanes obtained from the $n-m$ constraints in (4.k) replacing the inequality ' $\geqslant$ ' by ' $=$ ' contain the origin. However, this is not the case with respect to $\pi_{k}$.

We are going to study the case when the vertex of $C(\tau)$ belonging to the corresponding $\pi_{k}$ is one of those referred to in situation (a) (extreme points $A, B, C$ or $D$ ).

From Fig. 11(a) and (b), we can conclude that:

- If the vertex is $A$ or $B$, it is only necessary to study the constraints for which $\operatorname{sgn}\left[\lambda_{1}^{\prime}\left(w_{1 k}-w_{3 k}\right)\right]=$ $-\operatorname{sgn}\left[\lambda_{2}^{\prime}\left(w_{2 k}-w_{3 k}\right)\right]$, where the corresponding weight perturbation coefficients are such that $\phi_{1}=-\phi_{2}$.

- If the vertex is $C$ or $D$, it is only necessary to study the constraints for which $\operatorname{sgn}\left[\lambda_{1}^{\prime}\left(w_{1 k}-w_{3 k}\right)\right]=$ $\operatorname{sgn}\left[\lambda_{2}^{\prime}\left(w_{2 k}-w_{3 k}\right)\right]$, where the corresponding weight perturbation coefficients are such that $\phi_{1}=\phi_{2}$. 


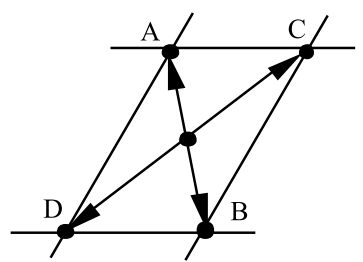

(a)

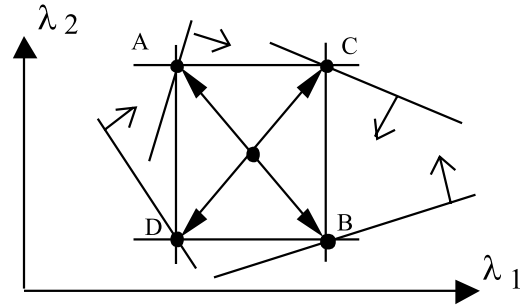

(b)

Fig. 11. (a) (on $\lambda_{1}-\lambda_{2}-\lambda_{3}$ ). (b) (on projection $\lambda_{1}-\lambda_{2}$ ).

For these extreme points $s_{r}=\operatorname{sgn}\left[\lambda_{r}^{\prime}\left(w_{r k}-w_{3 k}\right)\right], r=1,2, k=1, \ldots, n-m$.

For each of the $n-m$ constraints obtained from the reduced cost matrix the estimated weights and the extreme point of $C(\tau)$ belonging to $\pi_{k}$ under study are known. The distance between these two points as well as the $\tau_{k(\mathrm{a})}$ value can be easily determined.

These distances can be determined as in Section 2.2 (not being affected by the fact that they are on the plane $\left.\lambda_{1}+\lambda_{2}+\lambda_{3}=1\right)$.

Fig. 12 shows a 2-dimensional simplex in a Euclidean 3-dimensional weight space where $P=\hat{\lambda}=$ $\left(\hat{\lambda}_{1}, \hat{\lambda}_{2}, \hat{\lambda}_{3}\right)$ is the point corresponding to the estimated weights. The plane $\Pi_{k}$ is one of the planes obtained from the reduced cost matrix of a multiobjective simplex tableau associated with the corresponding basic efficient solution. $\Pi_{k}$ contains the origin and intersects $\Lambda$ in the boundary line $\pi_{k} . \overline{P X}$ is the perpendicular distance from $P$ to the plane $\Pi_{k}$ and can be determined by using $(8 . k)$.

$\overline{P T_{3(\mathrm{a})}}$ is the distance from $P$ to $\Pi_{k}$ along the direction of the vector $\vec{K}_{3(\mathrm{a})}=\left(-s_{1} \tau_{k(\mathrm{a})} \lambda_{1}^{\prime},-s_{2} \tau_{k(\mathrm{a})} \lambda_{2}^{\prime}\right.$, $\left.s_{1} \tau_{k(\mathrm{a})} \lambda_{1}^{\prime}+s_{2} \tau_{k(\mathrm{a})} \lambda_{2}^{\prime}\right)$.

$$
T_{3(\mathrm{a})}=\left(\hat{\lambda}_{1}-s_{1} \tau_{k(\mathrm{a})} \lambda_{1}^{\prime}, \hat{\lambda}_{2}-s_{2} \tau_{k(\mathrm{a})} \lambda_{2}^{\prime}, 1-\hat{\lambda}_{1}+s_{1} \tau_{k(\mathrm{a})} \lambda_{1}^{\prime}-\hat{\lambda}_{2}+s_{2} \tau_{k(\mathrm{a})} \lambda_{2}^{\prime}\right) \quad \text { and } \quad \overline{P T_{3(\mathrm{a})}}=\left|\vec{K}_{3(\mathrm{a})}\right| \text {. }
$$

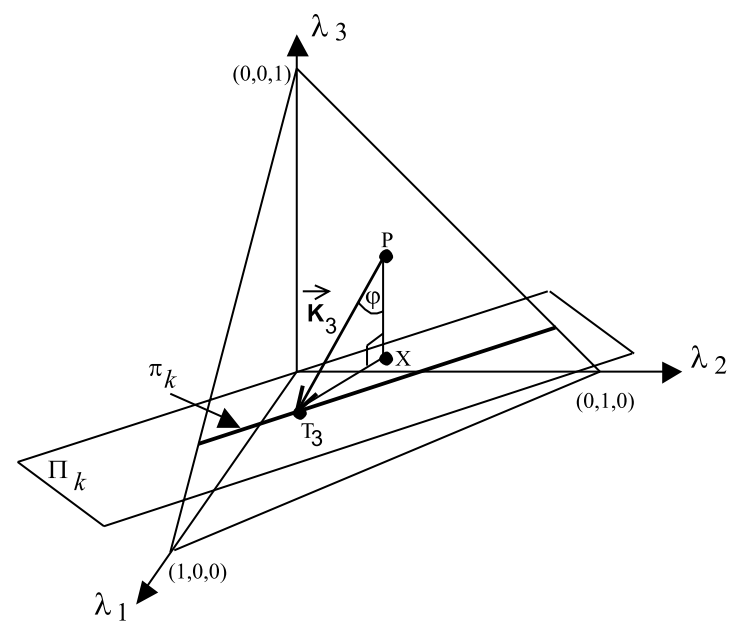

Fig. 12. 
Since $\overline{P T_{3(\mathrm{a})}}=\overline{P X} / \cos \varphi$ the corresponding $\tau_{k(\mathrm{a})}$ value is given by

$$
\tau_{k(\mathrm{a})}=\frac{\sum_{r=1}^{3} \hat{\lambda}_{r} w_{r k}}{\sum_{\substack{r \neq 1 \\ r \neq 3}}^{3}\left(-S_{r} \lambda_{r}^{\prime}\right)\left(-\left(w_{r k}-w_{3 k}\right)\right)}, \quad k=1, \ldots, n-m .
$$

Since we want to determine the smallest distance from the point to the plane, and the corresponding smallest $\tau_{k(\mathrm{a})}$, we have $s_{r}=\operatorname{sgn}\left(w_{r k}-w_{3 k}\right), r=1,2$, that is,

$$
\tau_{k(\mathrm{a})}=\frac{\sum_{r=1}^{3} \hat{\lambda}_{r} w_{r k}}{\sum_{\substack{r \neq 1 \\ r \neq 3}}^{3} \lambda_{r}^{\prime}\left|w_{r k}-w_{3 k}\right|}, \quad k=1, \ldots, n-m
$$

If $\tau_{k(\mathrm{a})}$ is finite then, for each $k=1, \ldots, n-m$, the $\phi_{r(\mathrm{a})}^{*}$ values can be obtained by

$$
\phi_{r(\mathrm{a})}^{*}=\tau_{k(\mathrm{a})} \operatorname{sgn}\left[\lambda_{r}^{\prime}\left(-\left(w_{r k}-w_{3 k}\right)\right)\right], \quad r=1,2 .
$$

If a similar study is performed when the vertex of $C(\tau)$ belonging to $\pi_{k}$ is one of those in situations (b) or (c), the following results are obtained.

When the vertex of $C(\tau)$ belonging to $\pi_{k}$ is one of those in situations (b) the maximum tolerance percentage of the objective function weights can be determined by

$$
\tau_{k(\mathrm{~b})}=\frac{\sum_{r=1}^{3} \hat{\lambda}_{r} w_{r k}}{\sum_{\substack{r \neq 1 \\ r \neq 2}}^{3} \lambda_{r}^{\prime}\left|w_{r k}-w_{2 k}\right|}, \quad k=1, \ldots, n-m
$$

If $\tau_{k(\mathrm{~b})}$ is finite then, for each $k=1, \ldots, n-m$, the $\phi_{r(\mathrm{~b})}^{*}$ values can be obtained by

$$
\phi_{r(\mathrm{~b})}^{*}=\tau_{k(\mathrm{~b})} \operatorname{sgn}\left[\lambda_{r}^{\prime}\left(-\left(w_{r k}-w_{2 k}\right)\right)\right], \quad r=1,3 .
$$

When the vertex of $C(\tau)$ belonging to $\pi_{k}$ is one of those in situation (c) the maximum tolerance percentage of the objective function weights can be determined by

$$
\tau_{k(\mathrm{c})}=\frac{\sum_{r=1}^{3} \hat{\lambda}_{r} w_{r k}}{\sum_{\substack{r=1 \\ r \neq 1}}^{3} \lambda_{r}^{\prime}\left|w_{r k}-w_{1 k}\right|}, \quad k=1, \ldots, n-m .
$$

If $\tau_{k(\mathrm{c})}$ is finite then, for each $k=1, \ldots, n-m$, the $\phi_{r(\mathrm{c})}^{*}$ values can be obtained by

$$
\phi_{r(\mathrm{c})}^{*}=\tau_{k(\mathrm{c})} \operatorname{sgn}\left[\lambda_{r}^{\prime}\left(-\left(w_{r k}-w_{1 k}\right)\right)\right], \quad r=2,3 .
$$

Let us consider an index, $q$, which takes the value 3 if the vertex of $C(\tau)$ is in situation (a), the value 2 for situation (b) and the value 1 for situation (c). Therefore, Eqs. (28.k)-(33.k) can be summarized as below.

The maximum tolerance percentage of the objective function weights can be determined by

$$
\tau_{k e}=\frac{\sum_{r=1}^{3} \hat{\lambda}_{r} w_{r k}}{\sum_{\substack{r=1 \\ r \neq q}}^{3} \lambda_{r}^{\prime}\left|w_{r k}-w_{q k}\right|}, \quad k=1, \ldots, n-m, \quad q=1,2,3, \quad e=(\mathrm{a}),(\mathrm{b}),(\mathrm{c}) .
$$

If $\tau_{k e}(e=(\mathrm{a}),(\mathrm{b}),(\mathrm{c}))$ is finite then, for each $k=1, \ldots, n-m$, the $\phi_{r e}^{*}$ values can be obtained by using:

$$
\phi_{r e}^{*}=\tau_{k e} \operatorname{sgn}\left[\lambda_{r}^{\prime}\left(-\left(w_{r k}-w_{q k}\right)\right)\right] ; \quad r=1,2,3, \quad q=1,2,3, \quad \text { and } \quad r \neq q .
$$

The $\tau^{*}$ value (possibly infinite) is determined by

$$
\tau^{*}=\operatorname{Min}_{\mathrm{e}=(\mathrm{a}),(\mathrm{b}),(\mathrm{c})}\left[\operatorname{Min}_{k \in K} \tau_{k e}\right] .
$$

As mentioned above, if the vertex of $C\left(\tau_{k e}\right)$ belonging to $\pi_{k}$ does not belong to $C\left(\tau_{k e}\right) \cap \Lambda$ it means that some vertex components are negative. 
For each index $y$ associated with a negative component another $C\left(\tau_{k e}\right)$ shall be sought such that one of the vertexes of $C\left(\tau_{k e}\right) \cap \Lambda$ is the point that results from the intersection of the corresponding $\pi_{k}$ with the plane $\lambda_{y}=0$. Herein, the values of $\tau_{k e}$ and $\phi_{r e}^{*}, k=1, \ldots, n-m, e=(\mathrm{a}),(\mathrm{b}),(\mathrm{c}) ; q=1,2,3 ; v=1,2,3$; $r \neq q$ and $y \neq q$ can be obtained by

$$
\tau_{k e}=\frac{\sum_{r=1}^{3} \hat{\lambda}_{r} w_{r k}+\phi_{y} \lambda_{y}^{\prime}\left(-\left(w_{y k}-w_{q k}\right)\right)}{\sum_{\substack{r \neq q ; r \neq y \\ r \neq y}}^{3} \lambda_{r}^{\prime}\left|w_{r k}-w_{q k}\right|},
$$

where $\phi_{y}=-100 \%$. If $\tau_{k e}(e=(\mathrm{a}),(\mathrm{b}),(\mathrm{c}))$ is finite then, for each $k=1, \ldots, n-m$,

$$
\phi_{r e}^{*}=\tau_{k e} \operatorname{sgn}\left[\lambda_{r}^{\prime}\left(-\left(w_{r k}-w_{q k}\right)\right)\right] .
$$

If some of the weight values are precise then all the procedure holds but the corresponding components in $\vec{K}_{3 e}$ become zero and they are $\hat{\lambda}_{r}$ in $T_{3 e}$.

\subsection{The weights are known to vary within intervals}

When $\Phi \neq \mathbb{R}^{3}$ and $\Phi=\Phi(\phi, \bar{\phi})$, the geometric analysis performed in Sections 2 and 3.1 can still be used.

The aim herein is to calculate the greatest value for the tolerance percentage of the objective function weights perturbation, $\tau^{*}$, for which the intersection of $\Phi$ with the corresponding $C\left(\tau^{*}\right) \cap \Lambda$ is a subset of region $H$ obtained with the weighting vector $\hat{\lambda}$. As in Section 3.1, the $\tau^{*}$ value is the smallest of all the three $\tau_{k e}$ values $(e=(\mathrm{a}),(\mathrm{b})(\mathrm{c}))$, and each one is also obtained as the smallest of the $n-m \tau_{k e}$ values found for $k=1, \ldots, n-m$.

By geometric considerations, it can be observed that if the region defined by $\Phi(\phi, \bar{\phi})$ is completely included in the hyperhalfspace defined by the $k$ constraint of $\left(4^{\prime} . k\right)$ then the $\tau_{k e}$ value is not finite.

Since $\lambda_{1}+\lambda_{2}+\lambda_{3}=1$, a simple procedure can be used to determine the three $\tau_{k e}$ values.

(1) For those indices $k$ for which $\tau_{k e}$ is finite the $\tau_{k e}$ values are initially computed by using the formula (for the unconstrained case $(34 . k))$

$$
\tau_{k e}=\frac{\sum_{r=1}^{3} \hat{\lambda}_{r} w_{r k}}{\sum_{r=1 ; r \neq q}^{3} \lambda_{r}^{\prime}\left|w_{r k}-w_{q k}\right|}, \quad q=1,2,3 .
$$

(2) For the index $h$ where the minimum value $\left(\tau_{k e h}\right)$ is attained, the $\phi_{r^{e h}}^{0}$ values are determined by

$$
\phi_{r^{e h}}^{0}=\operatorname{sgn}\left[\lambda_{r}^{\prime}\left(-\left(w_{r k}-w_{q k}\right)\right)\right] * \tau_{k e h}, \quad r=1,2,3, \quad q=1,2,3, \quad \text { and } \quad r \neq q .
$$

(3) Afterwards, the finite bounds $\phi_{r}$ and $\bar{\phi}_{r}$ are considered, and it is checked whether all constraints $\hat{\lambda}_{r} \geqslant \underline{\lambda}_{r}$ and $\hat{\lambda}_{r} \geqslant \bar{\lambda}_{r}, r=1,2,3$, are satisfied. In the positive case, the $\tau_{k e}$ value can be determined as the $\tau_{k e h}$. Otherwise, one of these constraints $(r=y)$ is not satisfied, and $\phi_{r^{t} h}^{t}$ must be re-computed by using

$$
\phi_{r^{e h}}^{t}= \begin{cases}0 & \text { if } \lambda_{r}^{\prime} w_{r k}=0, \\ \underline{\phi}_{r} & \text { if } \phi_{r}^{t-1}<\underline{\phi}_{r}, \\ \bar{\phi}_{r} & \text { if } \phi_{r}^{t-1}>\bar{\phi}_{r}, \\ \operatorname{sgn}\left[\lambda_{r}^{\prime}\left(-\left(w_{r k}-w_{q k}\right)\right)\right]\left(\frac{\sum_{r=1}^{3} \hat{\lambda}_{r} w_{r k}+\phi_{y}^{t-1} e h \lambda_{y}^{\prime}\left(-\left(w_{y k}-w_{q k}\right)\right)}{\sum_{r=1 ;: \neq q ; r \neq y}^{3} \lambda_{r}^{\prime}\left|w_{r k}-w_{q k}\right|}\right) & \text { otherwise. }\end{cases}
$$

Finally, $\tau_{k e h}$ is the smallest of all $\left|\phi_{r}^{t} e h\right|$. 
(4) The indices for which the current values of $\tau_{k e}$ are strictly less than the computed value for $\tau_{k e h}$ must be further analyzed. If there are no such indices then the maximum tolerance percentage $\tau_{k e}=\tau_{k e h}$ has been obtained. Otherwise, only those indices are considered and the procedure returns to step (3) to be performed a new iteration $t$.

Since negative weights have no meaning, if the vertex associated with $\tau_{k e}$ does not belong to $C(\tau) \cap \Phi \cap \Lambda$ (some components of the vertex are negative), it is necessary to perform a study similar to the one described in Section 3.1.

Although the above relaxation procedure has been presented as an iterative one (for the sake of further extension to $p>3$ ), for three objective function problems the $\tau_{k e}$ value, when finite, will be determined in at most 1 iteration ( $p-2$ iterations).

The proposed approach, incorporating the scaling condition, can easily be extended to the general case of more than three objective functions. However, the following issues must be taken into account.

The visual tools used in an interactive manner in our analysis can only be applied to three objective function problems. For $p>3$, the dialogue with the DM can become cumbersome and the dynamic analysis of the tolerance regions, which is one of the main features of our approach, can be undervalued. Thereafter, when considering $p$ objective functions it is necessary to separately study $p$ situations (cf. Section 3.1). Finally, if the vertex of $C\left(\tau_{k e}\right)$ belonging to $\pi_{k}$ does not belong to $C(\tau) \cap \Phi \cap \Lambda$ another $C\left(\tau_{k e}\right)$ shall be sought for each of the $p$ possible negative components (see Section 3 ).

\section{An illustrative example}

Let us consider the following linear problem with three objective functions (Hansen et al., 1989):

$$
\begin{aligned}
& \max z_{1}=10 x_{2}+80 x_{4}, \\
& \max z_{2}=10 x_{2}+10 x_{3}+20 x_{4}, \\
& \max z_{3}=10 x_{1}+10 x_{2}+10 x_{3}+10 x_{4},
\end{aligned}
$$

s.t.

$$
\begin{aligned}
& 4 x_{1}+9 x_{2}+7 x_{3}+10 x_{4} \leqslant 6000, \\
& x_{1}+x_{2}+3 x_{3}+40 x_{4} \leqslant 4000, \\
& x_{1}, x_{2}, x_{3}, x_{4} \geqslant 0
\end{aligned}
$$

$x_{5}$ and $x_{6}$ are slack variables of the constraints $(\boldsymbol{c} 1)$ and $(\boldsymbol{c} 2)$, respectively.

In the situation for which all basic efficient solutions are known, the graphs in Fig. 13(a) (indifference regions in $\Lambda$ ) and Fig. 13(b) (projection on $z_{1} z_{2}$ ) are displayed. These figures are actual copies of the screen presented to the user. Efficient solutions 1, 2 and 3 individually optimize objective functions $z_{1}, z_{2}$ and $z_{3}$, respectively. Solution 4 is the one studied in Hansen et al. (1989) obtained with $\lambda=(0.1 ; 0.3 ; 0.6)$. The characteristics of those solutions are shown in Table 1.

Considering the estimated weights $\hat{\lambda}=(0.1 ; 0.3 ; 0.6)$, leading to solution 4 , and $\Phi=\mathbb{R}^{3}$, the maximum tolerance value $\tau^{*}=25 \%$. This means that any variation of up to $25 \%$ in the estimated values of the weights does not change the efficient solution, as it can be concluded by analyzing Fig. 14. In Hansen et al. (1989) $\tau^{*}=21.7391 \%$. This difference derives from the normalizing conditions on the weights $\sum_{r=1}^{3} \lambda_{r}=1$, which nevertheless does not have influence on the computation of the efficient solution.

If the first weight is precise $\left(\hat{\lambda}_{1}=0.1\right)$, then $\tau^{*}=34.0136 \%$ (Fig. 15) which is greater than the value $25 \%$ previously obtained with no additional information and also greater than the value $23.8095 \%$ obtained in a 


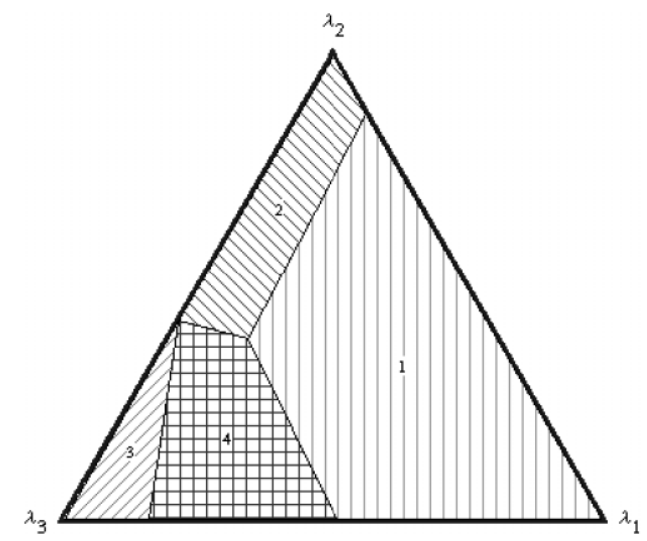

(a)

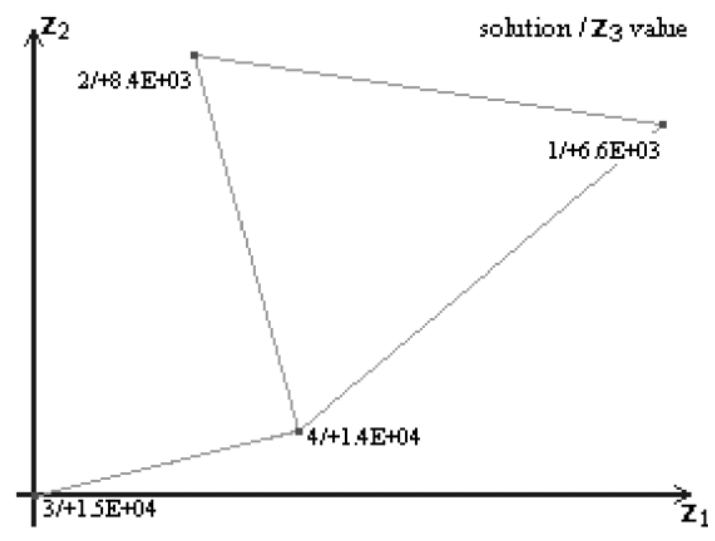

(b)

Fig. 13.

Table 1

Illustrative example basic efficient solutions

\begin{tabular}{lrrrrl}
\hline Solution & \multicolumn{1}{l}{$z_{1}$} & \multicolumn{1}{c}{$z_{2}$} & \multicolumn{1}{c}{$z_{3}$} & Area $(\%)$ & $\mathbf{x}_{\mathrm{B}}$ \\
\hline 1 & 12571.40 & 7428.57 & 6571.43 & 59.62 & $x_{2}=571.43 ; x_{4}=85.71$ \\
2 & 3200.00 & 8800.00 & 8400.00 & 14.33 & $x_{3}=800.0 ; x_{4}=40.0$ \\
3 & 0.00 & 0.00 & 15000.00 & 6.77 & $x_{1}=1500.0 ; x_{6}=2500.0$ \\
4 & 5333.33 & 1333.33 & 14000.00 & 19.28 & $x_{1}=1333.33 ; x_{4}=66.67$ \\
\hline
\end{tabular}

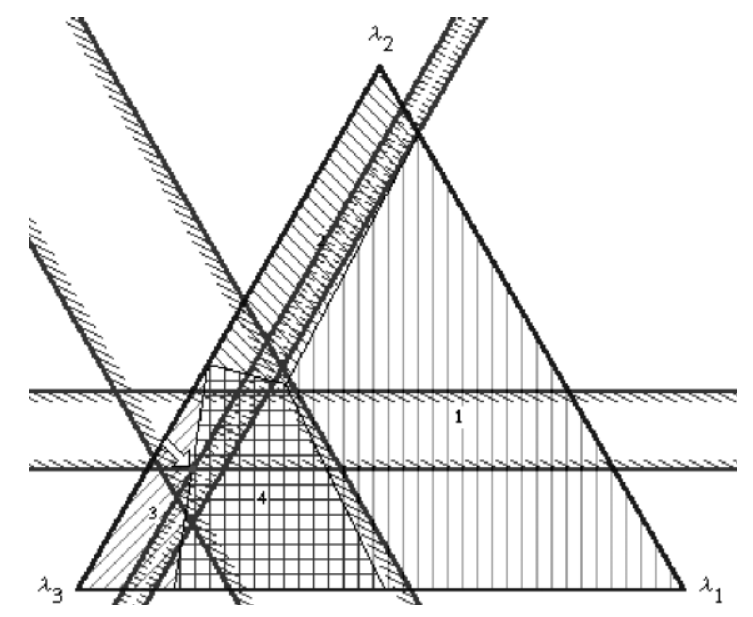

Fig. $14 . \hat{\lambda}=(0.1 ; 0.3 ; 0.6)$.

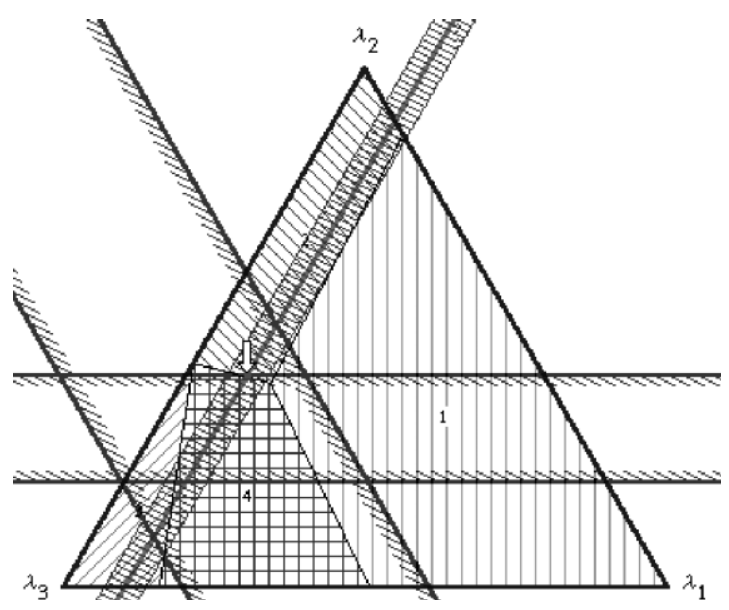

Fig. $15 . \hat{\lambda}=(0.1 ; 0.3 ; 0.6)$ and $\hat{\lambda}_{1}=0.1$.

similar situation in Hansen et al. (1989). That is, the second and the third weights can change simultaneously and independently within $34.0136 \%$ of their estimated values still maintaining the same (basic) efficient solution. 
Let us suppose that in addition to knowing $\hat{\lambda}_{1}$ precisely the DM knows that $\hat{\lambda}_{3}$ will not vary outside the interval $[0.5 ; 0.7]$. The intersection of $\Phi$ with $\Lambda$ is inside the indifference region corresponding to solution 4 ; therefore, the maximum tolerance of the weights is not finite (Fig. 16). As more a priori information concerning the variability of the weights becomes available, larger values for the maximum tolerance percentage could be obtained.

Note that if the DM considers that $\hat{\lambda}_{1}$ is not known with precision and $\hat{\lambda}_{3} \in[0.5 ; 0.7]$, then $\tau^{*}=25 \%$. Since the a priori information concerning the variability of the weights becomes more accurate, the maximum tolerance percentage value never decreases with respect to the unconstrained case. Fig. 17 shows the situation in which $\hat{\lambda}_{3} \in[0.5 ; 0.65]$ and the corresponding $\tau^{*}=34.2593 \%$.

If $\hat{\lambda}_{1}$ is precisely known and $\hat{\lambda}_{3} \geqslant 0.55$ then $\tau^{*}=47.619 \%$ (Fig. 18).

If $\hat{\lambda}_{1} \geqslant 0.095$ and $\hat{\lambda}_{3} \geqslant 0.55$ then a lower value for $\tau^{*}=43.0952 \%$ is obtained because $\hat{\lambda}_{1}$ is less accurate (Fig. 19).

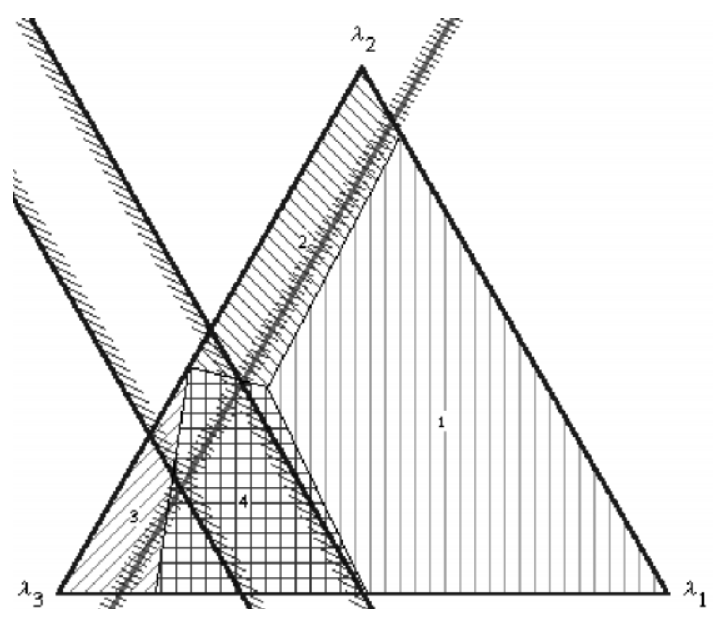

Fig. 16. $\hat{\lambda}=(0.1 ; 0.3 ; 0.6), \hat{\lambda}_{1}=0.1$ and $\hat{\lambda}_{3} \in[0.5 ; 0.7]$.

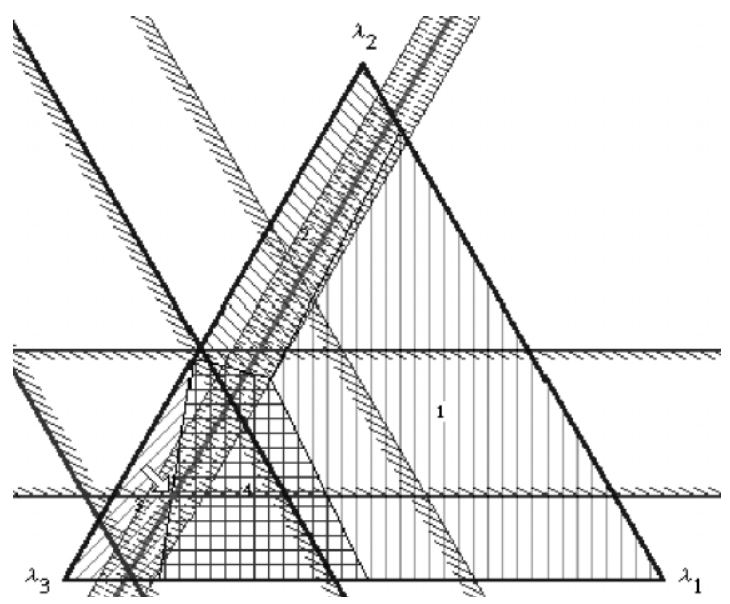

Fig. 18. $\hat{\lambda}=(0.1 ; 0.3 ; 0.6), \hat{\lambda}_{1}=0.1$ and $\hat{\lambda}_{3} \geqslant 0.55$.

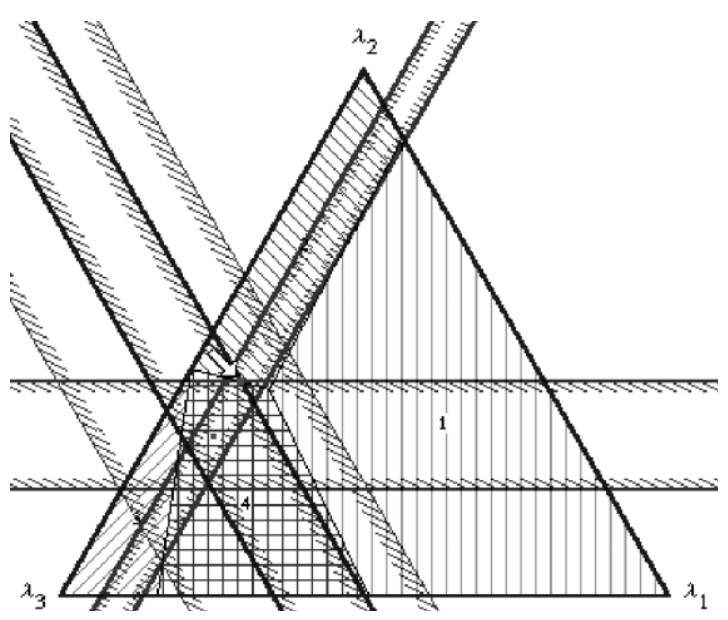

Fig. 17. $\hat{\lambda}=(0.1 ; 0.3 ; 0.6)$ and $\hat{\lambda}_{3} \in[0.5 ; 0.65]$.

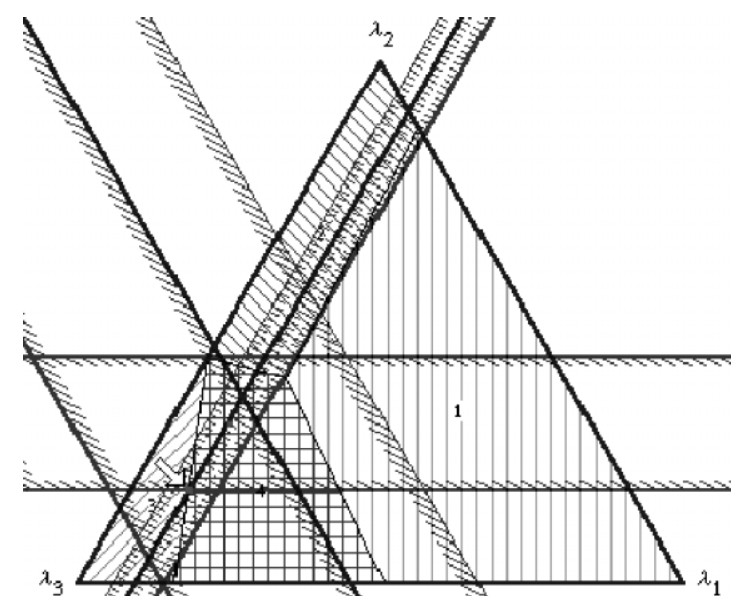

Fig. 19. $\hat{\lambda}=(0.1 ; 0.3 ; 0.6), \hat{\lambda}_{1} \geqslant 0.095$ and $\hat{\lambda}_{3} \geqslant 0.55$. 


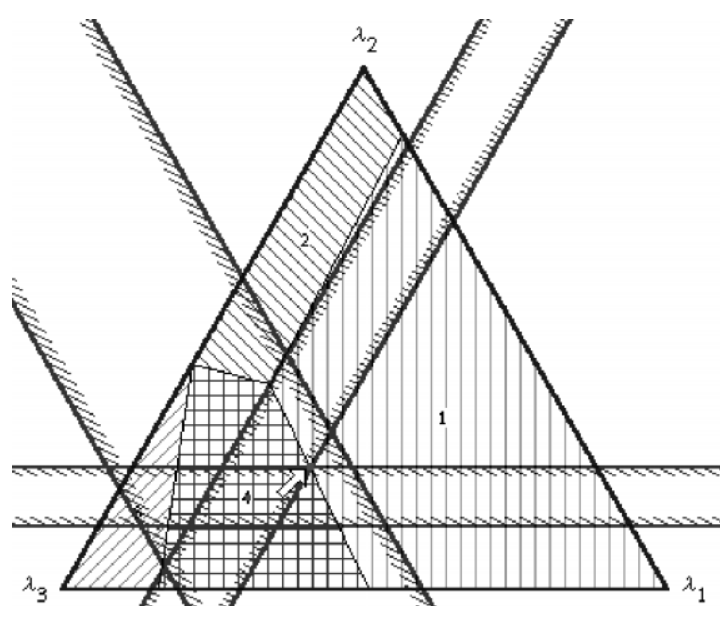

Fig. 20. $\hat{\lambda}=(0.2207 ; 0.1720 ; 0.6073)$.

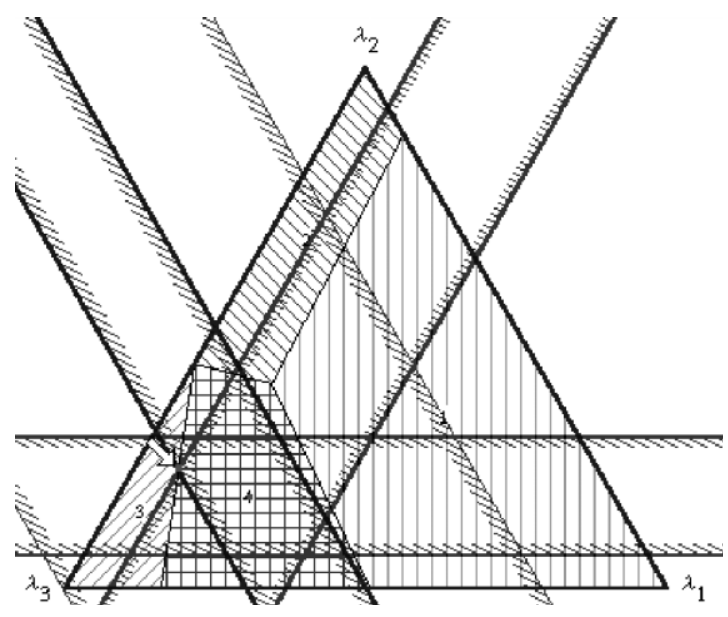

Fig. 21. $\hat{\lambda}=(0.2207 ; 0.1720 ; 0.6073)$ and $\hat{\lambda}_{3} \in[0.5 ; 0.7]$.

Let us suppose that the DM had considered the gravity center of the indifference region associated with solution $4, \hat{\lambda}=(0.2207 ; 0.1720 ; 0.6073)$, as the estimated weights. A similar analysis can be done. For example, if all the weights are not known with precision $\left(\Phi=\mathbb{R}^{3}\right) \tau^{*}=33.5252 \%$ (Fig. 20). If the DM considers $\hat{\lambda}_{3} \in[0.5 ; 0.7]$ then $\tau^{*}=66.0131 \%$ (Fig. 21). Both values are greater than $\tau^{*}=25 \%$ obtained under similar conditions for the very same solution computed with $\hat{\lambda}=(0.1 ; 0.3 ; 0.6)$.

Since the weights associated with the gravity center of the indifference region can be considered as the most stable for the corresponding basic efficient solution, then we would expect that the maximum tolerance values are not inferior to the values obtained with the previous weighting vector. However, if the nonnegativity conditions for the weights $\left(\lambda_{r} \geqslant 0, r=1,2,3\right)$ are binding on the boundary of the indifference region this is not always true. For instance, if solution 2 is studied with no additional information and considering as estimated weights $\hat{\lambda}=(0.055 ; 0.84 ; 0.105)$ then $\tau^{*}=132.108 \%$ (Fig. 22). However, if the gravity center of this indifference region $(\hat{\lambda}=(0.0667 ; 0.6670 ; 0.2663))$ is considered as estimated weights then $\tau^{*}=81.1464 \%$ (Fig. 23).

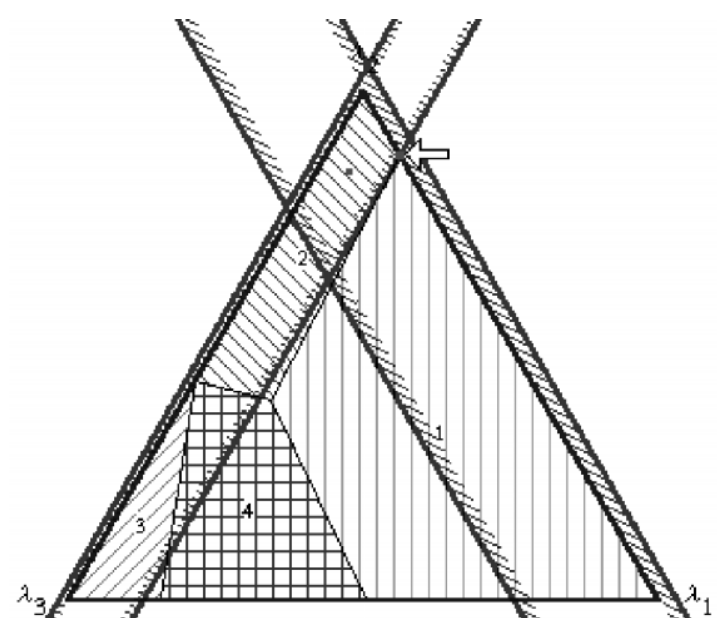

Fig. 22. $\hat{\lambda}=(0.055 ; 0.84 ; 0.105)$.

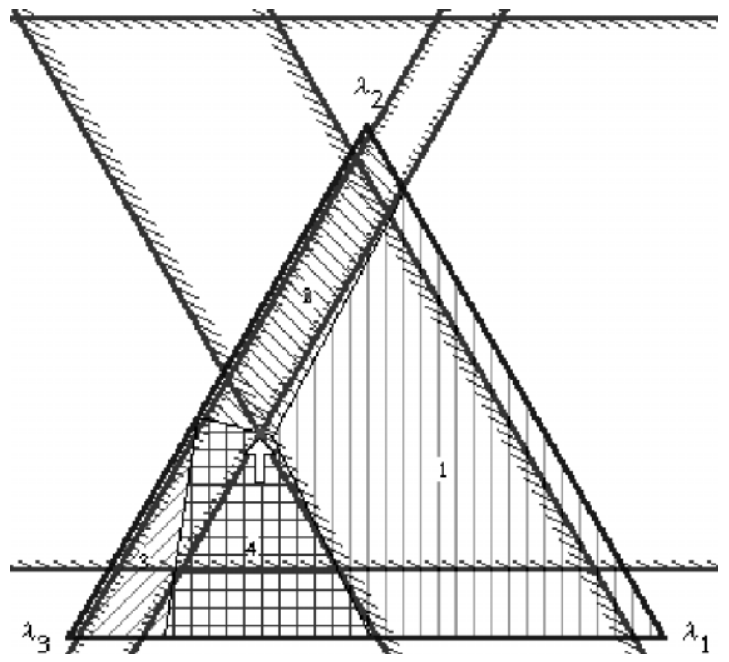

Fig. $23 . \hat{\lambda}=(0.0667 ; 0.6670 ; 0.2663)$. 


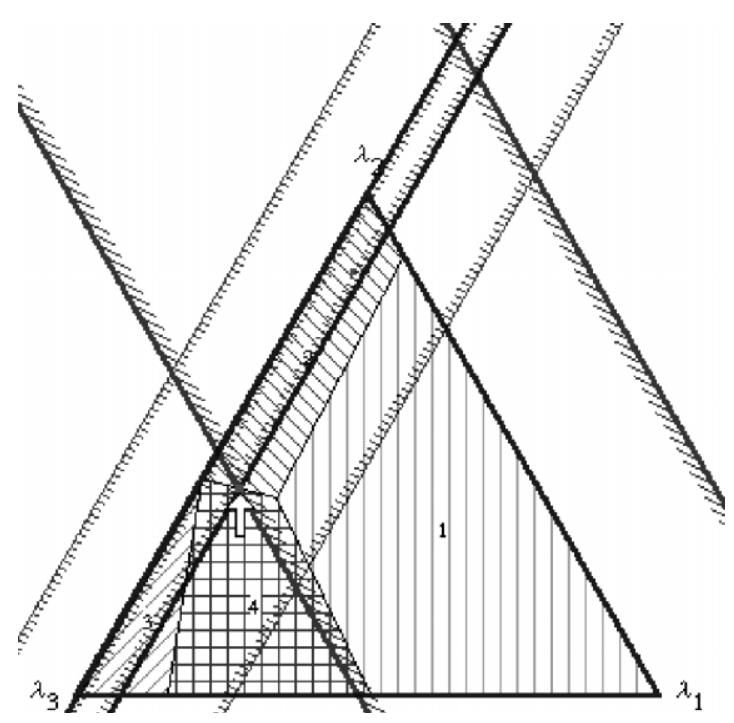

Fig. 24. $\hat{\lambda}=(0.055 ; 0.84 ; 0.105)$ and $\hat{\lambda}_{1} \in[0 ; 0.075]$.

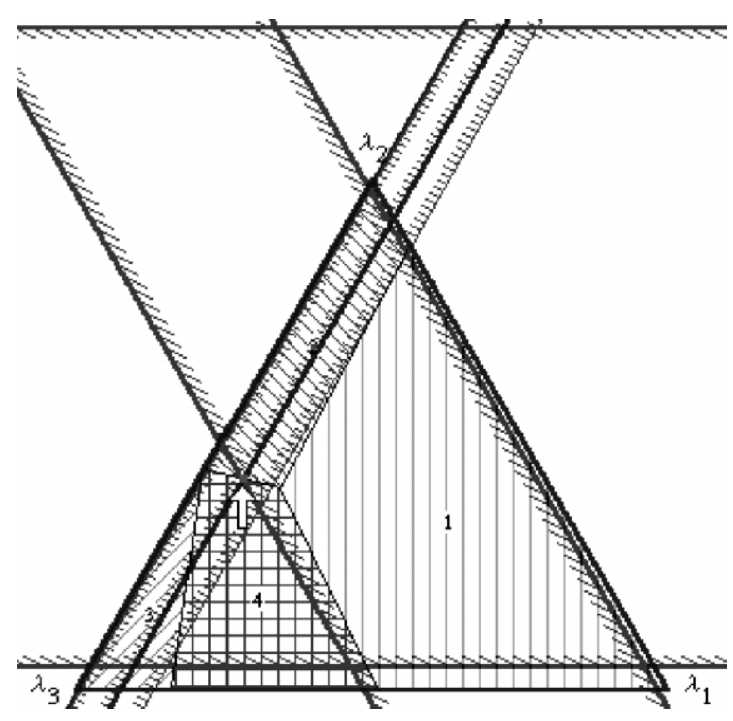

Fig. 25. $\hat{\lambda}=(0.0667 ; 0.6670 ; 0.2663)$ and $\hat{\lambda}_{1} \in[0 ; 0.075]$.

Since negative weights have no meaning the tolerance regions of the weight perturbations can be enlarged in order to obtain greater values for the maximum tolerance of the weights.

Similar conclusions can be obtained if $\Phi \neq \mathbb{R}^{3}$. For example, if the DM specifies that $\hat{\lambda}_{1} \in[0 ; 0.075]$ then $\tau^{*}=391.74 \%$ (for $\hat{\lambda}=(0.055 ; 0.84 ; 0.105)$ ) and $\tau^{*}=93.8958 \%$ (for the gravity center) (Figs. 24 and 25 , respectively).

If the DM studies solution 2 considering the estimated weights $\hat{\lambda}=(0.055 ; 0.84 ; 0.105)$ and $\hat{\lambda}_{2} \in[0 ; 0.85]$ (Fig. 26) then $\tau^{*}=133.766 \%\left(\tau^{*}=132.108 \%\right.$ was the value obtained with no a priori information). If additionally $\hat{\lambda}_{3} \geqslant 0.01$ (Fig. 27) then the tolerance regions of the weight perturbations can be enlarged and $\tau^{*}=139.845 \%$. Furthermore, if $\hat{\lambda}_{2} \in[0.8 ; 0.85]$ then the intersection of $\Phi$ with $\Lambda$ is a subset of the indifference region obtained with the weights $\hat{\lambda}$ and the maximum tolerance percentage is not finite (Fig. 28).

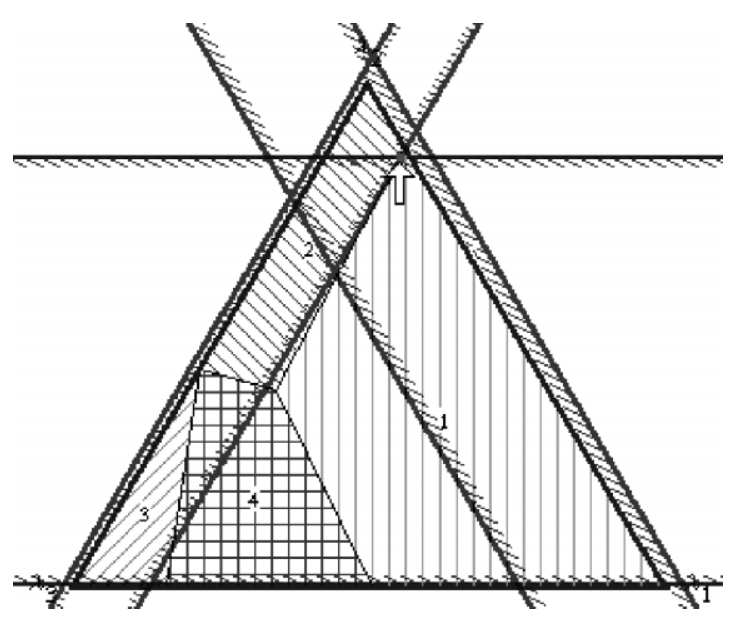

Fig. 26. $\hat{\lambda}=(0.055 ; 0.84 ; 0.105)$ and $\hat{\lambda}_{2} \in[0 ; 0.85]$.

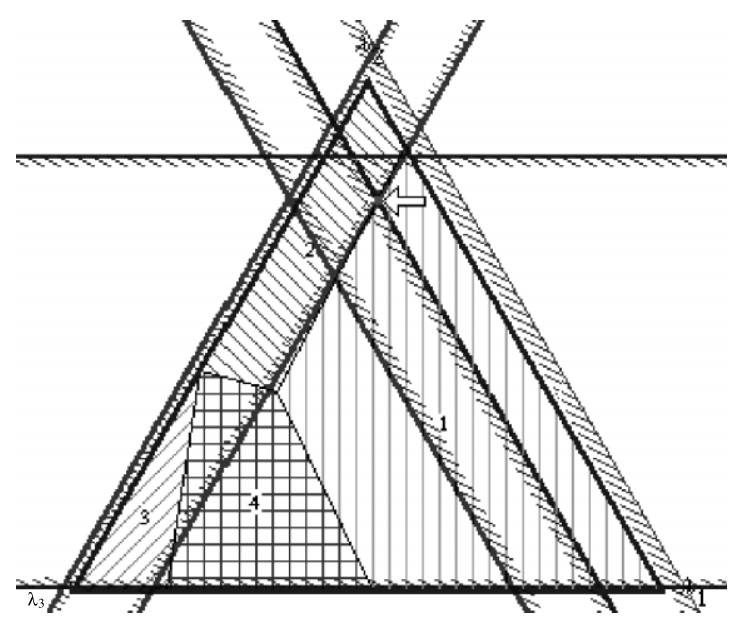

Fig. 27. $\hat{\lambda}=(0.055 ; 0.84 ; 0.105), \hat{\lambda}_{2} \in[0 ; 0.85]$ and $\hat{\lambda}_{3} \geqslant 0.01$. 


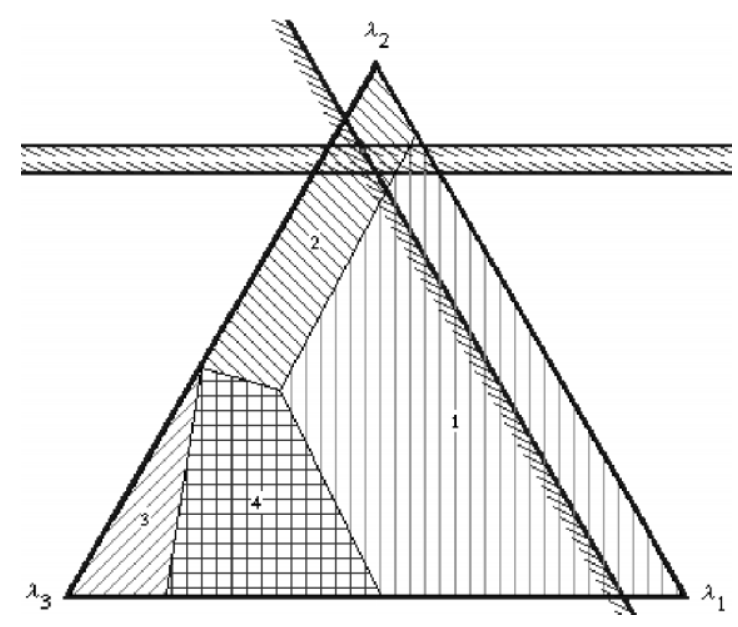

Fig. 28. $\hat{\lambda}=(0.055 ; 0.84 ; 0.105), \hat{\lambda}_{2} \in[0.8 ; 0.85]$ and $\hat{\lambda}_{3} \geqslant 0.01$.

\section{Conclusions}

A visual interactive tolerance approach to sensitivity analysis to deal with changes in the weighting vector of a MOLP problem has been presented. This approach is based on the analysis of the indifference regions corresponding to basic efficient solutions, which enables us to display graphical information interactively to the DM. It enables to visualize dynamically the changes of the tolerance region and study distinct sets of estimated weights as well as additional information.

This approach is suited to three-objective LP problems in order to make the most of the display of the indifference regions as a valuable tool to provide insightful information to the DM. The geometrical twodimensional simplex is used to display all relevant information in the same coherent graphic.

\section{References}

Antunes, C.H., Clímaco, J., 1992. Sensitivity analysis in MCDM using the weight space. Operations Research Letters 12, $187-196$.

Clímaco, J., Antunes, C.H., 1987. TRIMAP - an interactive tricriteria linear programming package. Foundations of Control Engineering 12, 101-119.

Clímaco, J., Antunes, C.H., 1989. Implementation of an user friendly software package - a guided tour of TRIMAP. Mathematical and Computer Modelling 12, 1299-1309.

Evans, J.R., 1984. Sensitivity analysis in decision theory. Decision Sciences 15, 239-247.

Hansen, P., Labbé, M., Wendell, R.E., 1989. Sensitivity analysis in multiple objective linear programming: The tolerance approach. European Journal of Operational Research 38, 63-69.

Mármol, A., Puerto, J., 1997. Special cases of the tolerance approach in multiobjective linear programming. European Journal of Operational Research 98, 610-616.

Ravi, N., Wendell, R.E., 1985. The tolerance approach to sensitivity analysis of matrix coefficients in linear programming: General perturbations. Journal of Operational Research Society 36, 943-950.

Ravi, N., Wendell, R.E., 1989. The tolerance approach to sensitivity analysis of matrix coefficients in linear programming. Management Science 35, 1106-1119.

Schneller, G.O., Sphicas, G.P., 1985. On sensitivity analysis in decision theory. Decision Sciences 16, 399-409. 
Wendell, R.E., 1984. Using bounds on the data in linear programming: The tolerance approach to sensitivity analysis. Mathematical Programming 29, 304-322.

Wendell, R.E., 1985. The tolerance approach to sensitivity analysis in linear programming. Management Science 31, 564-578.

Wondolowski, F.R., 1991. A generalization of Wendell's tolerance approach to sensitivity analysis in linear programming. Decision Sciences 22, 792-810. 